$10-2000$

\title{
Semiclassical Theory of Weighted Spectra for Regular Systems: Absorption Spectra and Decay Rates
}

\author{
M. W. Beims \\ William \& Mary \\ V. Kondratovich \\ William \& Mary \\ John B. Delos \\ William \& Mary, jbdelos@wm.edu
}

Follow this and additional works at: https://scholarworks.wm.edu/aspubs

Part of the Physics Commons

\section{Recommended Citation}

Beims, M. W.; Kondratovich, V.; and Delos, John B., Semiclassical Theory of Weighted Spectra for Regular Systems: Absorption Spectra and Decay Rates (2000). Physical Review A, 62(4).

https://doi.org/10.1103/PhysRevA.62.043401

This Article is brought to you for free and open access by the Arts and Sciences at W\&M ScholarWorks. It has been accepted for inclusion in Arts \& Sciences Articles by an authorized administrator of W\&M ScholarWorks. For more information, please contact scholarworks@wm.edu. 


\title{
Semiclassical theory of weighted spectra for regular systems: Absorption spectra and decay rates
}

\author{
M. W. Beims, ${ }^{*}$ V. Kondratovich, and J. B. Delos \\ Department of Physics, College of William and Mary, Williamsburg, Virginia 23187
}

(Received 12 January 2000; revised manuscript received 17 May 2000; published 7 September 2000)

\begin{abstract}
We derive a simple semiclassical representation to describe the large-scale structure of the spectrum of regular systems weighted by some arbitrary function $W$. Examples of weighted spectra are the width-weighted spectrum, which represents the decay rate of an unstable system, and the oscillator-strength-weighted spectrum, which represents the photoabsorption rate. Semiclassical representations of such spectra involve stationary-phase contributions, which are periodic or closed orbits, and end-point contributions, which are loops on an extremal torus. The theory provides the link between semiquantal formulas and the closed-orbit theory of atomic photoabsorption. It also allows calculation of an average decay rate without knowledge of the widths of individual quantum states.

PACS number(s): 32.80.Rm, 03.65.Sq, 32.70.Cs, 32.60.+i
\end{abstract}

\section{WEIGHTED SPECTRA: INTRODUCTION}

We consider a quantum system that has energy levels $E_{j}$, and for each quantum state there is some observable quantity $W_{j}$. This quantity may be $f_{j}$, the oscillator strength for transition from some specified initial state to a level $j ; \Gamma_{j}$, the width of the $j$ resonance; $\Delta E_{j}$, the splitting between a pair of states labeled $j$ due to tunneling through a barrier; or $1 / \Gamma_{j}$, the lifetime of the state $j$.

We define the weighted spectrum as

$$
D \mathcal{W}(E)=\frac{d \mathcal{W}(E)}{d E}=\sum_{j} W_{j} \delta\left(E-E_{j}\right) .
$$

Each state with energy $E=E_{j}$ is weighted by the quantity $W_{j}$. The integrated weight $\mathcal{W}(E)$ is defined as

$$
\mathcal{W}(E)=\int_{-\infty}^{E} D \mathcal{W}\left(E^{\prime}\right) d E^{\prime}=\sum_{\left\{j \mid E_{j}<E\right\}} W_{j} .
$$

The purpose of this paper is to find semiclassical representations of the spectrum (1) or (2) for regular systems.

What is the meaning of such weighted spectra, and what is the use of semiclassical approximations to them? (1) If all the weights are set to unity, $W_{j}=1$, then Eq. (1) represents the density of states. Gutzwiller [1] showed that the semiclassical approximation for the density of states involves a sum over classical periodic orbits (PO's) of the system. Short classical orbits are connected with the large-scale structure of the density of states, while longer orbits give higher resolution detail. Thus the semiclassical approximation gives a way to obtain the density of states averaged over an energy interval without examining individual states. (2) The weights $W_{j}$ could be set to $f_{j}^{(i)}$, the oscillator strength for a transition from some selected initial state $i$ to state $j$. Then Eq. (1) represents the oscillator-strength density. For transitions between well-localized initial states and large Rydberg states, the semiclassical representation involves a sum over classical

\footnotetext{
* Present address: Universidade Federal do Parana, Departamento de Fisica, Caixa Postal 19081, 81531-990 Curitiba - PR, Brazil.
}

orbits that are closed at the atomic nucleus [2]. (3) In symmetric double-well systems, the weights could be the splitting $\Delta E_{j}$ between a nearly degenerate pair of states labeled $j$. Creagh and Whelan [3] showed that the semiclassical representation involves complex orbits that tunnel through the barrier. (4) If a particle can tunnel from quasibound to free states (as for example in the Stark effect) then the weight $W_{j}$ could be defined either as the width $\Gamma_{j}$ of the quasibound state or as the lifetime $\hbar / \Gamma_{j}$. The width-weighted spectrum represents the decay rate of unstable systems as a function of energy. In an earlier paper [4], we asserted that (for regular systems) the corresponding representation involved a sum over irreducible loops on the "extremal torus." That formula will be derived in this paper.

Recently Bogomolny and Rouben [5] and Saraga and Monteiro [6] have used a width-weighted spectrum in an important application. In a "resonant tunneling diode," electrons tunnel through a potential barrier, move quasiclassically in the presence of applied electric and magnetic fields, and then tunnel through a second barrier to escape. The measured current as a function of applied fields shows fluctuations, some but not all of which seem to be correlated with periodic orbits. Theoretical considerations show that the current is proportional to tunneling matrix elements averaged over a range of quantum states. Therefore the measured current is related to a kind of width-weighted spectrum. Theory [5] and calculations [6] indicate that in this case the semiclassical representation involves a sum over nonperiodic complex orbits called saddle orbits.

In all the above cases, the semiclassical approximation gives simple representations of averaged properties, largescale structure, or short-time behavior of the system. For example, a semiclassical representation of a width-weighted spectrum may give the average decay rate of an unstable system over a range of energies, even if individual energies and widths are not known. In a few cases $[7,8]$ the orbit sum can be extended far enough that properties of individual quantum states can also be calculated; this is the case in the present paper.

We consider in this paper only "regular" systems, in which classical orbits form tori and in which quantum states can be accurately calculated by quantization of action vari- 
ables. For such systems, Berry and Tabor [7] showed how to connect the Einstein-Brillouin-Keller (EBK) quantization scheme with the Gutzwiller periodic orbit sum. They represented the density of states as a certain sum of integrals over action variables, and they showed that each stationary-phase point of one of these integrals corresponds to a rational torus (a family of periodic orbits). End-point contributions to these integrals also entered the theory; in their case these end points represented isolated stable periodic orbits (O-points on a surface of section).

Our analysis is similar to theirs. We find that for the width-weighted spectrum, one end point provides the dominant contribution and this end point is the "extremal torus." For the oscillator-strength density, stationary-phase approximations dominate, and these are closed orbits.

\section{AN INTEGRAL REPRESENTATION OF A REGULAR WEIGHTED SPECTRUM}

\section{A. Regular spectrum}

A quantum system has a regular spectrum if the classical motion is bounded and integrable: the particles are restricted to a finite region of configuration space, there exist as many isolating integrals of motion as the number of degrees of freedom, the system has a full set of action variables $\mathbf{J}$ $=\left(J_{1}, J_{2}, \ldots, J_{d}\right)$ (where $d$ is the number of degrees of freedom), and it is possible to write the classical Hamiltonian as a function of these action variables, $H=H(\mathbf{J})$. In the semiclassical approximation, these action variables are quantized according to the EBK condition

$$
\mathbf{J}_{\mathbf{n}}=2 \pi \hbar(\mathbf{n}+\boldsymbol{\mu})
$$

where the $i$ th component $\mu_{i}$ of the vector $\boldsymbol{\mu}$ is $1 / 4$ of the Maslov index for a cycle of the corresponding coordinate. The label $j$ for each quantum state is equivalent to this collection of quantum numbers

$$
j=\mathbf{n}=\left(n_{1}, n_{2}, \ldots, n_{d}\right),
$$

and the discrete energy levels are values of the Hamiltonian $H(\mathbf{J})$ evaluated at the quantized actions,

$$
E_{j} \equiv E_{\mathbf{n}}=H\left(J_{\mathbf{n}}\right)=H[2 \pi \hbar(\mathbf{n}+\boldsymbol{\mu})],
$$

so that the energy levels are discrete values of a continuous function. We assume that the weights $W_{j}$ are also discrete values of a continuous function of the action variables

$$
W_{j}=W_{\mathbf{n}}=W\left(J_{\mathbf{n}}\right)=W[2 \pi \hbar(\mathbf{n}+\boldsymbol{\mu})] .
$$

\section{B. Restricting the weights to some portion of the spectrum}

Experimental data are usually restricted to a finite range of the energy, and therefore we want to restrict the weighted spectrum to the same portion of the spectrum. This can be introduced here by letting the weight function be the product of two factors,

$$
W(\mathbf{J})=W_{\text {range }}(\mathbf{J}) W_{\text {physics }}(\mathbf{J}) .
$$

$W_{\text {physics }}$ is the physical quantity that we want to study. $W_{\text {range }}$ is a function that picks out the observed or the relevant part of the spectrum; this can be a step function which is equal to one if $E_{a}<E<E_{b}$, and zero otherwise. Alternatively it might be a smoother cutoff function that has the effect of restricting the energy to some physically relevant range. When we study width-weighted spectra, we may choose $W_{\text {range }}$ so that it selects only narrow "below barrier" resonances, and eliminates broad "above-barrier" resonances. Restriction to a finite range also helps to avoid questions about convergence.

We write the formulas below for the case that

$$
W_{\text {range }}(\mathbf{J})= \begin{cases}1, & E_{a} \leqslant H(\mathbf{J}) \leqslant E_{b} \\ 0 & \text { otherwise, }\end{cases}
$$

remembering that other choices are also possible. Then Eq. (2) is replaced by

$$
\mathcal{W}(E)=\sum_{\left\{\mathbf{n} \mid E_{a}<E<E_{b}\right\}} W_{\mathbf{n}} .
$$

Now we apply $\mathcal{W}(E)$ to regular systems by using relation (6) in Eq. (9)

$$
\mathcal{W}(E)=\sum_{\left\{\mathbf{n} \mid E_{a}<E<E_{b}\right\}} W\left[J_{\mathbf{n}}=2 \pi \hbar(\mathbf{n}+\boldsymbol{\mu})\right],
$$

and transform into an integral representation over the action variables

$$
\begin{aligned}
\mathcal{W}(E) & =\int_{E_{a}<E<E_{b}} d \mathbf{J} W(\mathbf{J}) \sum_{\mathbf{n}} \delta[\mathbf{J}-2 \pi \hbar(\mathbf{n}+\boldsymbol{\mu})], \\
& =\frac{1}{(2 \pi \hbar)^{d}} \int_{E_{a}<E<E_{b}} d \mathbf{J} W(\mathbf{J}) \sum_{\mathbf{n}} \delta\left[\frac{\mathbf{J}}{2 \pi \hbar}-(\mathbf{n}+\boldsymbol{\mu})\right] .
\end{aligned}
$$

The restricted sum in Eq. (10) has been made into an unrestricted sum in Eq. (11); the cutoff function $W_{\text {range }}(E)$ now carries the restrictions. This allows us to use the Poisson sum formula as in [7] for the sum over the $\delta$ functions in Eq. (11).

\section{Using the Poisson sum formula}

Let $g(\mathbf{n})$ be discrete values of a continuous function $g(\mathbf{x})$, and let $G(\mathbf{s})$ be the Fourier transform of $g(\mathbf{x})$,

$$
G(\mathbf{s})=\int e^{2 \pi i \mathbf{s}} g(\mathbf{x}) d \mathbf{x} .
$$

The Poisson sum formula asserts that the sum of $g(\mathbf{x})$ evaluated on the integers $\mathbf{n}$ is the sum of $G(\mathbf{s})$ evaluated on the integers $\mathbf{M}$, i.e.,

$$
\sum_{\mathbf{n}} g(\mathbf{n})=\sum_{\mathbf{M}} G(\mathbf{M}) .
$$

We use this to evaluate $\mathcal{W}(E)$ by setting 


$$
g(\mathbf{x})=\delta\left(\frac{\mathbf{J}}{2 \pi \hbar}-\boldsymbol{\mu}-\mathbf{x}\right)
$$

from which

$$
G(\mathbf{M})=e^{2 \pi i \mathbf{M} \cdot[\mathbf{J} /(2 \pi \hbar)-\boldsymbol{\mu}]} .
$$

By using these relations, Eq. (11) can be written as

$$
\mathcal{W}(E)=\frac{1}{(2 \pi \hbar)^{d}} \sum_{\mathbf{M}} e^{-2 \pi i \mathbf{M} \cdot \boldsymbol{\mu}} \int d \mathbf{J} W(\mathbf{J}) e^{i \mathbf{M} \cdot \mathbf{J} / \hbar} .
$$

The sum over $\mathbf{M}$ includes all positive and negative integers, including zero.

\section{Curvilinear coordinates in action space}

In the next step (again following [7]) we assume that over the relevant range we can define curvilinear coordinates in action space such that $E(\mathbf{J})$ is one coordinate. All the other $(d-1)$ coordinates are denoted $\boldsymbol{\beta}(\mathbf{J})$. For systems with a regular spectrum, all the coordinates $\boldsymbol{\beta}(\mathbf{J})$ can be associated with the conserved quantities of the integrable system. We also take $\boldsymbol{\beta}$ to be dimensionless, but otherwise we leave it unspecified for now. Transforming to curvilinear coordinates, Eq. (16) becomes

$$
\begin{aligned}
& \mathcal{W}(E)=\frac{1}{(2 \pi \hbar)^{d}} \sum_{\mathbf{M}} e^{-2 \pi i \mathbf{M} \cdot \boldsymbol{\mu}} \int d E d \boldsymbol{\beta} W(E, \boldsymbol{\beta}) \\
& \times\left|\frac{\partial(\mathbf{J})}{\partial(E, \boldsymbol{\beta})}\right| e^{i \mathbf{M} \cdot \mathbf{J}(E) / \hbar},
\end{aligned}
$$

and finally, Eq. (1) expressed as a function of the action variables is given by

$$
\begin{aligned}
D \mathcal{W}(E)= & \frac{1}{(2 \pi \hbar)^{d}} \sum_{\mathbf{M}} e^{-2 \pi i \mathbf{M} \cdot \boldsymbol{\mu}} \int d \boldsymbol{\beta} W(E, \boldsymbol{\beta}) \\
& \times\left|\frac{\partial(\mathbf{J})}{\partial(E, \boldsymbol{\beta})}\right| e^{i \mathbf{M} \cdot \mathbf{J}(E) / \hbar}
\end{aligned}
$$

where $\partial(\mathbf{J}) / \partial(E, \boldsymbol{\beta})$ is the Jacobian of transformation between the action variables and the conserved quantities. The formula (18) is a general expression for the spectrum (1) weighted by some observable quantity $W_{j}$. Note that in Eq. (18), the weight $W(E, \boldsymbol{\beta})$ appears as a continuous function of $E$ and $\boldsymbol{\beta}$ and not as the discrete function $W_{j}$.

The term $\mathbf{M}=0$ in Eq. (18) represents the approximation of replacing the sum in Eq. (1) by an integral, and can be treated as a "background," slowly varying in energy. The terms $\mathbf{M} \neq 0$ give oscillatory corrections to this background. This will be shown later in more detail for the example considered in this paper.

The method used to calculate the integral over $\boldsymbol{\beta}$ in Eq. (18) depends upon the specific physical situation to be considered and upon the relevant weight function $W(E, \boldsymbol{\beta})$. In general, however, the integral is rapidly oscillatory for small $\hbar$, and the main contributions come from stationary phase points and the boundaries of the $\boldsymbol{\beta}$ domain. Contributions of stationary phase points will be discussed in Sec. IV, while contributions of end points will be discussed in Sec. III.

\section{WIDTH-WEIGHTED SPECTRUM}

In this section we show in detail the derivation and results for the width-weighted spectrum in the Stark problem defined in a previous work [4]. Additional results for the below-barrier case are also shown.

Consider a system having a particle bound in a potential well, and separated from free motion by a potential-energy barrier. Because of tunneling, the states of the particle in the well are quasibound, with complex energies $\mathcal{E}_{j}=E_{j}-i \Gamma_{j} / 2$, where $\Gamma_{j}$ is the width, which is related to the decay time of the state by $\tau_{j}=\hbar / \Gamma_{j}$. By choosing the weight function to be $W_{j}=\Gamma_{j}$, the weighted spectrum (1) is now the "widthweighted spectrum', given by [4]

$$
D \Gamma(E)=\sum_{j} \Gamma_{j} \delta\left(E-E_{j}\right)
$$

Each quasibound state with real energy $E=E_{j}$ is weighted by the width $\Gamma_{j}$.

As an example, we consider in this paper the case of an electron subjected simultaneously to a Coulomb and an electric field (the Stark problem). In this case, the electron is bound to the nucleus by the Coulomb potential, but the external electric field creates a potential barrier through which the electron can escape by tunneling.

We start in this section by giving some information about the classical motion and quantum spectrum in this system. This will be done in Sec. III A. In Sec. III B we give the quantization condition which includes tunneling effects and we give the formula to calculate the widths. Some numerical results for the "semiquantal", width-weighted spectrum are also shown. Finally, in the last part of this section (Sec. III C), the semiclassical formulas for the width-weighted spectrum are derived and compared numerically with the semiquantal results.

The final result of this section is: the Fourier-transform of the scaled width-weighted spectrum has peaks at actions coresponding to loops on the "external torus," the last torus before escape over the barrier; the heights of these peaks are related to canonical periods of motion, including the imaginary "period" of underbarrier motion.

\section{A. The hydrogen atom in an electric field}

The Hamiltonian of the hydrogen atom in an electric field is (using atomic units $\hbar=1, e=1, m_{e}=1$ )

$$
H=\frac{\mathbf{p}^{2}}{2}+\mathcal{V}(\rho, z)=E,
$$

where

$$
\mathcal{V}(\rho, z)=-\frac{1}{\left(\rho^{2}+z^{2}\right)^{1 / 2}}+F z
$$

$F$ is the strength of the applied electric field and $E$ is the total energy. For simplicity, we consider the cylindrically symmetric states, $L_{z}=m \hbar=0$. The scaled version of the Hamiltonian (20) is given by 

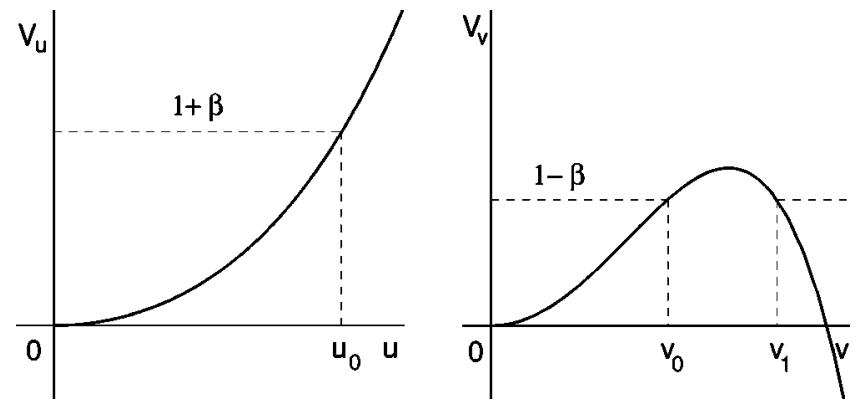

FIG. 1. Effective potential energy of the Hamiltonians $H_{u}$ and $H_{v}$ from Eq. (23) (arbitrary units). $(1+\beta)$ and $(1-\beta)$ are, respectively, the effective energies in the $u$ and $v$ motions. The points $v_{0}$ and $v_{1}$ represent the turning points for the underbarrier motion.

$$
\widetilde{H}=\frac{\tilde{\mathbf{p}}^{2}}{2}-\frac{1}{\left(\tilde{\rho}^{2}+\tilde{z}^{2}\right)^{1 / 2}}+\tilde{z}=\varepsilon,
$$

where we used the scaled variables, $w=F^{-1 / 4}, \tilde{\mathbf{q}}=w^{-2} \mathbf{q}$, $\tilde{\mathbf{p}}=w \mathbf{p}$, and the scaled energy $\varepsilon=E / F^{1 / 2}$. The classical behavior of the system depends only on one parameter, the scaled energy.

The Stark Hamiltonian (22) is separable in semiparabolic coordinates $(u=\sqrt{\tilde{r}+\tilde{z}}, v=\sqrt{\tilde{r}-\tilde{z}})$, and the corresponding effective Hamiltonians are

$$
\begin{aligned}
& H_{u}=p_{u}^{2} / 2-\varepsilon u^{2}+u^{4} / 2=(1+\beta), \\
& H_{v}=p_{v}^{2} / 2-\varepsilon v^{2}-v^{4} / 2=(1-\beta),
\end{aligned}
$$

where $\beta$ is the separation constant, which is related to the angle of ejection of the electron from the atom by $\beta=\cos \theta$, where $\theta$ is defined relative to the $+z$ axis. This separation constant can be identified as the $z$ component of the generalized Runge-Lenz vector in the presence of an electric field [9].

From the form of Eq. (23), we see that we can define "effective potential energies"

$$
\begin{aligned}
& V_{u}(u)=-\varepsilon u^{2}+u^{4} / 2, \\
& V_{v}(v)=-\varepsilon v^{2}-v^{4} / 2,
\end{aligned}
$$

and that the right-hand side (rhs) $(1 \pm \beta)$ can be regarded as an "effective energy" associated with $u$ or $v$ motion. These quantities are shown in Fig. 1. If $\beta=-1$, the effective energy in the $v$ motion is 2 and in the $u$ motion is 0 . This means that all the motion is in the $v$ coordinate, or in the "downhill" direction (i.e., in the $-z$ direction). For $\beta=1$ exactly the opposite occurs: the motion is totally in the $u$ coordinate, or the "uphill" direction (the $+z$ direction). For values of $\beta$ between -1 and +1 , there is motion in both $u$ and $v$ coordinates.

There is a saddle point in the real potential energy $\mathcal{V}(\rho, z)$ at the energy $E=-2 F^{1 / 2}(\varepsilon=-2)$, and below this energy, classical motion in the $(u, v)$ coordinates is bound. However, because there is an effective (dynamical) barrier in the $v$ motion which allows the possibility of tunneling (see Fig. 1),

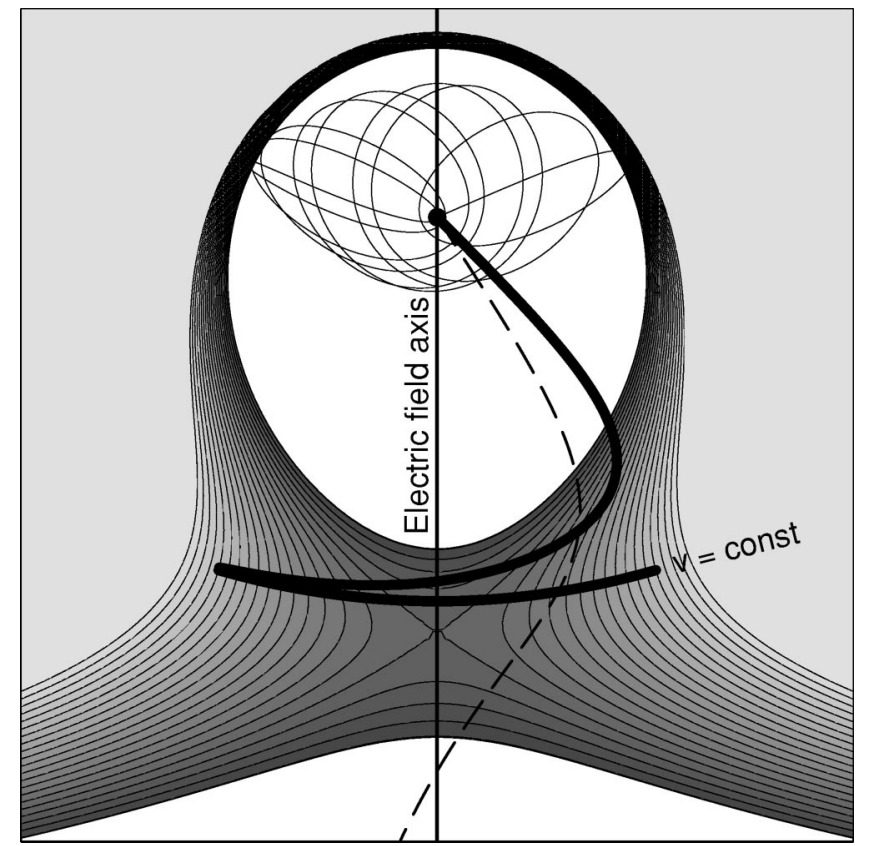

FIG. 2. Electron orbits in the $\tilde{z} \tilde{\rho}$ plane superimposed on a slice of the potential-energy landscape $\widetilde{V}(\tilde{z}, \tilde{\rho})$ for $-2.05 \leqslant \widetilde{V} \leqslant-1.8$. The size of of the figure is $2 \times 2$ in the scaled units. The regions with $\widetilde{V}<-2.05$ are unshaded; the region with $\widetilde{V}>-1.8$ has a uniformly light shade. The level contours are provided in steps of 0.0025 . The orbit launched at the critical angle $\theta_{c}$ approaches an unstable periodic orbit above the potential saddle (bold line). All orbits having this limit lie on the extremal torus. For ejection angles less than the critical angle $\theta_{c}$ the orbit remains bound (thin line). For larger ejection angles it goes over the effective barrier and escapes (dashed line).

the quantum spectrum is quasidiscrete, with states of long lifetime and quite sharply defined energy. High-Rydberg states of the pure Coulomb field are split into regular Stark manifolds.

For energies above the saddle but below the zero-field ionization threshold, i.e., for $-2<\varepsilon<0$, the $u$ motion is still bound but the classical $v$ motion may be bound or free, depending on the value of $\beta$. The critical $\beta_{c}$ which separates bound from unbound motion is $\beta_{c}=\cos \theta_{c}=\left(1-\varepsilon^{2} / 2\right)$. For $\beta=\beta_{c}$ the effective energy $\left(1-\beta_{c}\right)$ of $H_{v}$ is located exactly at the top of the effective barrier shown in Fig. 1. There is an unstable PO, for which $v$ sits on top of the barrier while $u$ oscillates, and there are orbits approaching or receding from this PO. For these, the corresponding period in $v$ motion diverges. Figure 2 shows examples of trajectories that start with different ejection angles. Trajectories are shown in the $(\tilde{\rho}, \tilde{z})$ space for $\varepsilon=-1.85$. The thin solid line shows a bound trajectory with ejection angle less than the critical angle $\theta_{c}$, while the dashed line shows an escaping trajectory with an angle larger then $\theta_{c}$. The bold line is the trajectory with an ejection angle equal to the critical angle, and it approaches the unstable PO, which lies on a segment of a parabola $v$ $=$ const. This orbit hangs near the top of the $v$ barrier and it lies on what we call here an "extremal torus" or "last surviving torus," just before the trajectories escape over the 
barrier.This torus is the set of all such orbits with any relative phase between the $u$ motion and the $v$ motion.

The coexistence of bound and unbound classical motion for $-2<\varepsilon<0$ is manifested in the quantum system in the following way: in this range of $\varepsilon$ the absorption spectrum consists of quasidiscrete levels superposed on a smoothly rising continuum. The higher-energy (uphill) quasidiscrete levels of each manifold survive, while the lower-energy (downhill) levels in each manifold are broadened into the smooth continuum [10].

\section{B. "Semiquantal"' results}

For regular systems, the EBK quantization of action variables (3) can be used to find the discrete energies. If we neglect tunneling through the potential-energy barrier, then quantization of action variables with fixed field strength $F$ leads to a discrete real set of eigenvalues $\left(E_{\mathbf{n}}, \beta_{\mathbf{n}}\right)$; the corresponding trajectories are called "eigentrajectories" or "eigentori," labeled by quantum numbers $\mathbf{n}=\left(n_{u}, n_{v}\right)$ [11]. (Eigentrajectories are not to be confused with classical periodic or closed orbits.)

When we also take into account tunneling through the barrier, the quantization conditions become complex. To calculate the width of a quasibound state $\Gamma_{\mathbf{n}}$, or the associated tunneling rate $\Gamma_{\mathbf{n}} / \hbar$, we fix the electric field $F$ and allow the energy $E$ and separation constant $\beta$ to become complex.

In the present case it is convenient to consider a scaled spectrum. Neglecting tunneling, we fix the scaled energy $\varepsilon$ $=E w^{2}$, and find quantized values of $w_{\mathbf{n}}$ and $\beta_{\mathbf{n}}$. Let us define the scaled width-weighted spectrum by setting a value of $\varepsilon$, calculating such a set of $w_{\mathbf{n}}$ 's, and then weighting them by the energy-width as follows:

$$
D \Gamma=\sum_{\mathbf{n}} \Gamma_{\mathbf{n}} \delta\left(E-E_{\mathbf{n}}\right)=\sum_{\mathbf{n}} \frac{\widetilde{\Gamma}_{\mathbf{n}}}{(-2 \varepsilon)} \delta\left(w-w_{\mathbf{n}}\right),
$$

where $\widetilde{\Gamma}_{\mathbf{n}}=\Gamma_{\mathbf{n}} w^{3}[12]$.

We use semiclassical approximations to determine $w_{\mathbf{n}}$ and $\Gamma_{n}$, but, since the focus of this calculation is on the individual quantum states, we call the results of this section "semiquantal" results. In this way we distinguish these results from the "semiclassical" results in Sec. III C. Those results focus on short trajectories and on the average properties of the spectrum.

Quantization conditions for a system having one degree of freedom were given by Child [13]. We have a separable system with two degrees of freedom, with tunneling possible in only one coordinate, so the quantization conditions for the scaled spectrum are

$J_{u}(\varepsilon, \beta ; w)=w \widetilde{J}_{u}(\varepsilon, \beta)=2 \pi\left(n_{u}+\frac{1}{2}\right)$,

$J_{v}(\varepsilon, \beta ; w)=w \widetilde{J}_{v}(\varepsilon, \beta)=2 \pi\left(n_{v}+\frac{1}{2}\right)-\frac{i}{2} \ln \left(1+e^{-K}\right)-\delta$.

Here $\widetilde{J}_{u}, \widetilde{J}_{v}$ are the scaled actions

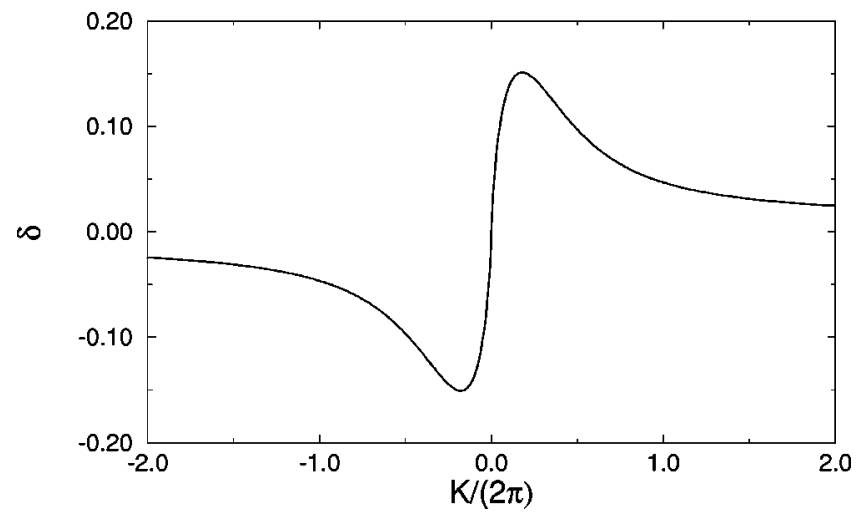

FIG. 3. Parabolic-barrier correction $\delta$ vs $K /(2 \pi)$ (atomic units). $\delta$ is never greater than 0.15 .

$$
\begin{aligned}
& \widetilde{J}_{u}(\varepsilon, \beta)=\int_{o s c} p_{u} d u=2 \int_{0}^{u_{0}} \sqrt{2(1+\beta)+2 \varepsilon u^{2}-u^{4}} d u, \\
& \widetilde{J}_{v}(\varepsilon, \beta)=\int_{o s c} p_{v} d v=2 \int_{0}^{v_{0}} \sqrt{2(1-\beta)+2 \varepsilon v^{2}+v^{4}} d u,
\end{aligned}
$$

and $K$ is the action integral (see also Fig. 1)

$$
K=2 \int_{v_{0}}^{v_{1}}\left|p_{v}\right| d v
$$

for a "full cycle" of underbarrier motion in the $v$ coordinate. If the energies of the tunneling states are not too close to the top of the barrier, then the imaginary term in Eq. (26) can be replaced by

$$
-\frac{i}{2} \ln \left(1+e^{-K}\right) \approx-\frac{i}{2} e^{-K} .
$$

We used this approximation in our calculations. The expression (26), which is uniform near the barrier top, can be treated in a similar way (see Appendix A).

The quantum numbers $n_{u}=0,1,2, \ldots$ and $n_{v}$ $=0,1,2, \ldots$ in Eq. (26) are integers which define the parabolic states and

$$
\delta=\arg \Gamma\left[\frac{1}{2}+i \frac{K}{2 \pi}\right]-\frac{K}{2 \pi} \log \left(\frac{|K|}{2 \pi}\right)+\frac{K}{2 \pi},
$$

is the parabolic-barrier correction. This correction is often small, and in most cases it does not significantly affect the eigenvalues. A graph of $\delta$ vs $K / 2 \pi$ is shown in Fig. 3. We see that $\delta$ is never greater than about 0.15 . Since in Eq. (26) it is being combined with integers $n_{v}$ times $2 \pi$, we conclude that it affects the location of eigenvalues by at most $0.15 / 2 \pi \rightarrow 2.4 \%$ of their spacing. This is large compared to their width, but small compared to their spacing, so $\delta$ can often be neglected in these calculations. (On the other hand, we will see that it cannot at all be neglected in the semiclassical formulas given in the following section.)

To calculate $w_{\mathbf{n}}$ 's, we pick a value of $\varepsilon$, and throw away the imaginary term $(-i / 2) \exp (-K)$ in Eq. (26). To calculate 
the energy widths, we see that if they are not too large, they can be determined from Eq. (26) by the formula

$$
\Gamma_{\mathbf{n}}=\frac{\frac{\partial J_{u}}{\partial \beta}}{\frac{\partial\left(J_{u}, J_{v}\right)}{\partial(E, \beta)}} e^{-K}=\frac{\frac{\partial \widetilde{J}_{u}}{\partial \beta}}{w^{3} \frac{\partial\left(\widetilde{J}_{u}, \widetilde{J}_{v}\right)}{\partial(\varepsilon, \beta)}} e^{-w \widetilde{K}}=\frac{\widetilde{\Gamma}_{\mathbf{n}}}{w^{3}},
$$

where $\partial\left(J_{u}, J_{v}\right) / \partial(E, \beta)$ is the Jacobian of the transformation from action variables $\left(J_{u}, J_{v}\right)$ to the conserved quantities $(E, \beta)$. In a one-dimensional system, the corresponding formula for $\Gamma$ is the vibrational frequency times an exponential factor. Our system is separable, and tunneling is only in the $v$ coordinate, but it is not equivalent to a onedimensional system. The tunneling terms make both $E$ and $\beta$ complex in such a way that $J_{u}$ stays real. Therefore the preexponential factor in Eq. (30) involves both $J_{u}$ and $J_{v}$.

Finally, in this calculation, we include all resonances which lie below the effective barrier in the $v$ coordinate; thus we include all quasidiscrete states, but we do not include above-barrier resonances, which may be so broad that they might better be regarded as background continuum. Our justification for omitting above-barrier resonances is that in Eq. (7) we can apply weights to the states in any way we choose, and we choose to give above-barrier resonances zero weight (we want a measure of tunneling rates).

\section{Below saddle}

First we look at a spectrum for $\varepsilon=-2.1$. This is below the saddle in the real potential energy $\mathcal{V}(\rho, z)$, so all states are quasidiscrete. A width-weighted spectrum is shown in Fig. 4(a). Each point of this figure marks the width $\log \Gamma_{\mathbf{n}}$ plotted against its quantized $w_{\mathbf{n}}$. For example at principal quantum number $n=n_{u}+n_{v}+1=7$, we see states with $u, v$ quantum numbers from $\left(n_{u}=6, n_{v}=0\right)$ (the most "uphill" and longest-lived state of the group) to $\left(n_{u}=0, n_{v}=6\right)$ (the most "downhill" and shortest-lived state). The other states labeled in Fig. 4(a) are for $n=15$. Each state also has a quantized value of $\beta_{\mathbf{n}}$; those states with the largest width (i.e., larger $n_{v}$ ), have $\beta_{\mathbf{n}}$ closer to -1 . As we expected, when $\beta_{\mathbf{n}}$ is closer to -1 , there is more $v$ motion, and therefore the probability of tunneling is larger (see the discussion near Fig. 1). Such states are the most important in a widthweighted spectrum [14].

If we try to plot $\widetilde{\Gamma}_{\mathbf{n}}$ from Eq. (30) itself as a function of $w$, we will see something which decays with an overall factor $\exp [-w \widetilde{K}]$, where $\widetilde{K}=K / w$ is the scaled action of underbarrier motion. In Fig. 4(b) we extracted this overall factor and plotted $\widetilde{\Gamma}_{\mathbf{n}} / \exp [-w \widetilde{K}]$. The result looks like a periodic sequence of widths vs $w$, and by comparing with Fig. 4(a), we observe that by this linear plot, we extracted only those states with the biggest widths, i.e., $n_{u}=0$.

\section{Above saddle}

Now let us examine a case with energy above the saddle of $V(\rho, z)$. As explained earlier, the classical $v$ motion can

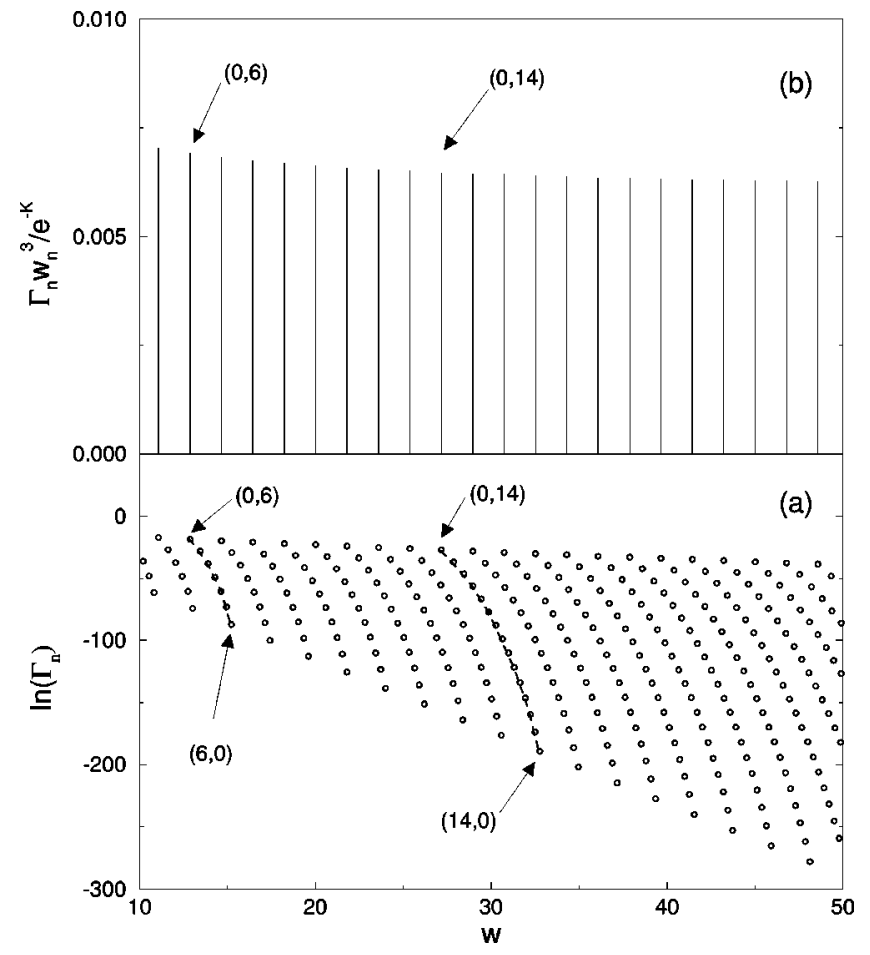

FIG. 4. Semiquantal result of (a) $\ln \Gamma_{\mathbf{n}}$ and (b) $\widetilde{\Gamma}_{\mathbf{n}} / e^{-w \tilde{K}}$ vs $w$ for the scaled energy $\varepsilon=-2.1$ (atomic units). The parabolic quantum numbers $\left(n_{u}, n_{v}\right)$ are labeled for the most downhill $(0,6),(0,14)$ and in (b) for the most uphill $(6,0),(14,0)$ states for the principal quantum numbers $n=7$ and $n=15$. We see that the peaks in (b) are located at the position of the most downhill states.

be bound or free, and we examine only quasidiscrete states below the top of the effective potential-energy barrier (Fig. 1). Figure 5(a) shows an example of a scaled quasidiscrete spectrum for a scaled energy $(\varepsilon=-1.5)$ well above the saddle (at this scaled energy $\beta_{c}=-0.125, \theta_{c} \simeq 97$ degrees). Again each point of this figure marks the width $\log \Gamma_{n}$ plotted against its quantized $w_{n}$. Again some Stark manifolds are marked. Now the most downhill states do not have $n_{u}=0$ and $n_{v}=n-1$-their corresponding orbits go right over the effective barrier. Instead we have for example $\left(n_{u}=3, n_{v}\right.$ $=5)$ or $\left(n_{u}=8, n_{v}=13\right)$ as the most downhill states of the $n=9$ or $n=22$ manifolds, respectively.

In Fig. 5(b), $\widetilde{\Gamma}_{\mathbf{n}}$ is plotted as a function of $w$ and we see that in this case the result looks like a quasiperiodic sequence of widths vs $w$.

\section{Semiclassical results}

As stated earlier, we call the above results "semiquantal." Let us now obtain a "semiclassical" formula starting from Eq. (18). Let us rewrite Eq. (18) for the case of the Stark problem. Here $d=2$, the vector $\mathbf{M}$ is a two-component vector, $\mathbf{M}=\left(M_{u}, M_{v}\right)$ and the Maslov vector is $\boldsymbol{\mu}$ $=(1 / 2,1 / 2)$, because the motion in each coordinate has a Maslov index of 2. The semiclassical expression for the width-weighted spectrum is then given from Eq. (18) by 


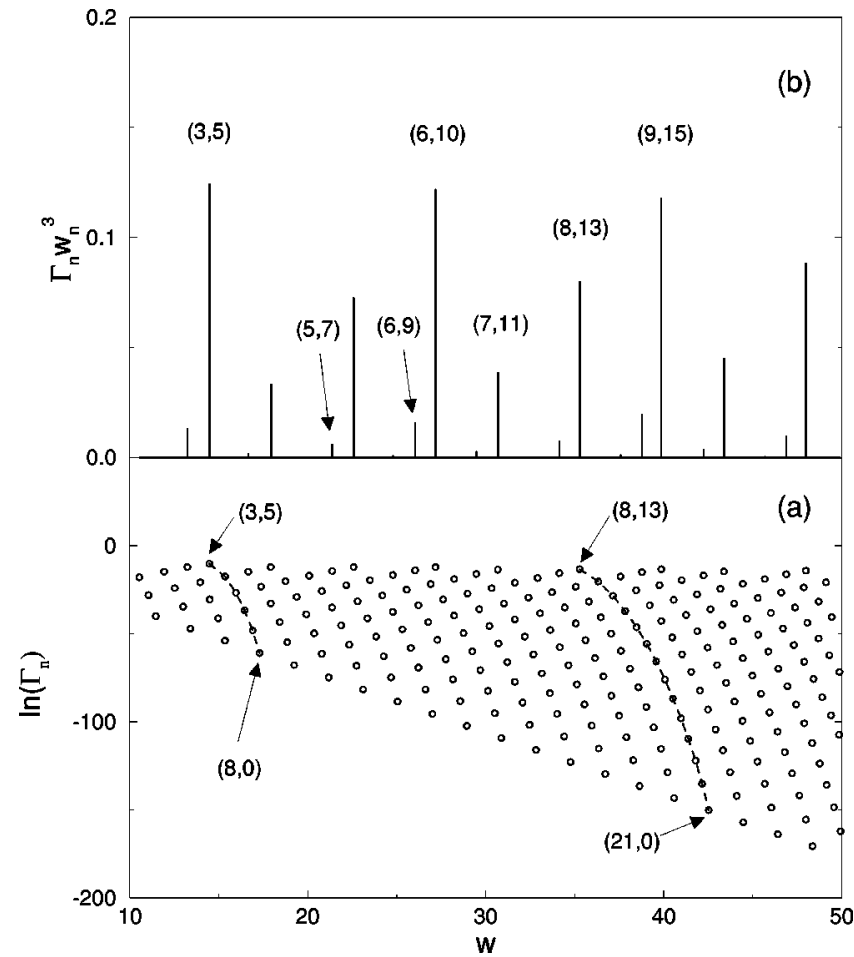

FIG. 5. Semiquantal result of (a) $\ln \Gamma_{\mathbf{n}}$ and (b) $\widetilde{\Gamma}_{\mathbf{n}}$ vs $w$ for the scaled energy $\varepsilon=-1.5$ (atomic units). The parabolic quantum numbers $\left(n_{u}, n_{v}\right)$ are labeled in (a) for the most downhill $(3,5),(8,13)$ and for the most uphill $(8,0),(21,0)$ states for the principal quantum numbers $n=9$ and $n=22$. Some quantum numbers $\left(n_{u}, n_{v}\right)$ are also shown in (b), and it is clear to see that large widths correspond to the most downhill states.

$$
\begin{aligned}
D \Gamma(E)= & \frac{1}{(2 \pi)^{2}} \sum_{M_{u}, M_{v}}(-1)^{\left(M_{u}+M_{v}\right)} \int_{\beta_{c}(\varepsilon)}^{1} d \beta \Gamma(E, \beta) \\
& \times\left|\frac{\partial\left(J_{u}, J_{v}\right)}{\partial(E, \beta)}\right| e^{i\left[M_{u} J_{u}(E, \beta)+M_{v} J_{v}(E, \beta)\right]}
\end{aligned}
$$

Here $\Gamma(E, \beta)$ is given by the same expression as $\Gamma_{\mathbf{n}}$ from Eq. (30), but it is considered as a continuous function of $E$ and $\beta$. The lower limit of integration $\beta_{c}(\varepsilon)$ is $\beta_{c}(\varepsilon)=-1$ for $\varepsilon$ $<-2$ (below saddle), or $\beta_{c}(\varepsilon)=1-\varepsilon^{2}$ for $-2<\varepsilon<0$ (above saddle).

The widths are greatest for the "downhill" states, having $\beta$ close to $\beta_{c}(\varepsilon)$. As $\beta$ increases, the underbarrier action integral (28) increases, and the exponential factor $\exp [-K(E, \beta)]$ in $\Gamma(E, \beta)$ [Eq. (30)] decreases rapidly. Therefore, for a width-weighted spectrum, we expect that the main contribution to the integral over $\beta$ comes from the vicinity of the end point $\beta_{c}(\varepsilon)$.

Let us therefore expand

$$
\begin{gathered}
\widetilde{J}_{u}(\varepsilon, \beta) \simeq \hat{J}_{u}+\frac{1}{2} \hat{\tau}_{u}(\varepsilon)\left[\beta-\beta_{c}(\varepsilon)\right], \\
\widetilde{J}_{v}(\varepsilon, \beta)-\frac{\delta}{w} \simeq \hat{J}_{v}-\frac{1}{2} \hat{\tau}_{v}(\varepsilon, w)\left[\beta-\beta_{c}(\varepsilon)\right],
\end{gathered}
$$

$$
\widetilde{K}(\varepsilon, \beta) \simeq \hat{K}+\frac{1}{2} \hat{\tau}_{K}(\varepsilon)\left[\beta-\beta_{c}(\varepsilon)\right]
$$

with $\hat{J}_{u \text { or } v}=\widetilde{J}_{u \text { or } v}\left(\epsilon, \beta_{c}(\epsilon)\right)$, and

$$
\begin{gathered}
\hat{\tau}_{u}=2 \frac{\partial \widetilde{J}_{u}}{\partial \beta}, \quad \hat{\tau}_{K}=2 \frac{\partial \widetilde{K}}{\partial \beta}, \\
\hat{\tau}_{v}=-2 \frac{\partial\left(\widetilde{J}_{v}+\delta / w\right)}{\partial \beta} .
\end{gathered}
$$

All quantities here must be evaluated at $\beta=\beta_{c}(\varepsilon)$. These quantities $\hat{\tau}$ are called "canonical periods." Evaluation of the integral in Eq. (31) then gives

$$
D \Gamma=e^{-w \hat{K}} \sum_{M_{u}, M_{v}} D_{M_{u}, M_{v}} e^{i w\left(M_{u} \hat{J}_{u}+M_{v} \hat{J}_{v}\right)}
$$

where

$$
D_{M_{u}, M_{v}}=\frac{1}{4 \pi^{2}} \frac{(-)^{M_{u}+M_{v}} \hat{\tau}_{u}}{\left[\hat{\tau}_{K}-i\left(M_{u} \hat{\tau}_{u}-M_{v} \hat{\tau}_{v}\right)\right]} .
$$

Equation (34) is a double Fourier sum of terms that oscillate as a function of $w$ with angular frequencies $\left(M_{u} \hat{J}_{u}\right.$ $\left.+M_{v} \hat{J}_{v}\right)$. These are action integrals for $M_{u}$ cycles of $u$ motion and $M_{v}$ cycles of $v$ motion, i.e., they are action integrals associated with irreducible loops of the extremal torus, the one with $\beta=\beta_{c}(\varepsilon)$. All positive, negative, and zero values of $M_{u}$ and $M_{v}$ are included in the sum.

\section{Below saddle}

In this section we will discuss the results from Eq. (34) for scaled energy $\varepsilon<-2$. In this case, the end point $\beta_{c}(\varepsilon)$ $=-1$, so the effective energy in $H_{u}$ is zero and there is no motion in the $u$ coordinate, i.e., $J_{u}=0$. Here the extremal torus degenerates to the downhill periodic orbit lying on the $v$ axis (or the negative $z$ axis). In this case the sum over $M_{u}$ can be evaluated using

$$
\sum_{M_{u}} \frac{(-)^{M_{u}}}{x-M_{u} \pi}=\frac{1}{\sin x}
$$

where

$$
x=\left(\pi M_{v} \hat{\tau}_{v} / \hat{\tau}_{u}-i \pi \hat{\tau}_{K} / \hat{\tau}_{u}\right) .
$$

Equation (34) is then given by

$$
D \Gamma=e^{-w \hat{K}} \sum_{M_{v}} D_{M_{v}} e^{i w M_{v} \hat{J}_{v}}
$$

where

$$
D_{M_{v}}=\frac{-i}{4 \pi} \frac{(-)^{M_{v}}}{\sin \left(\pi M_{v} \hat{\tau}_{v} / \hat{\tau}_{u}-i \pi \hat{\tau}_{K} / \hat{\tau}_{u}\right)} .
$$




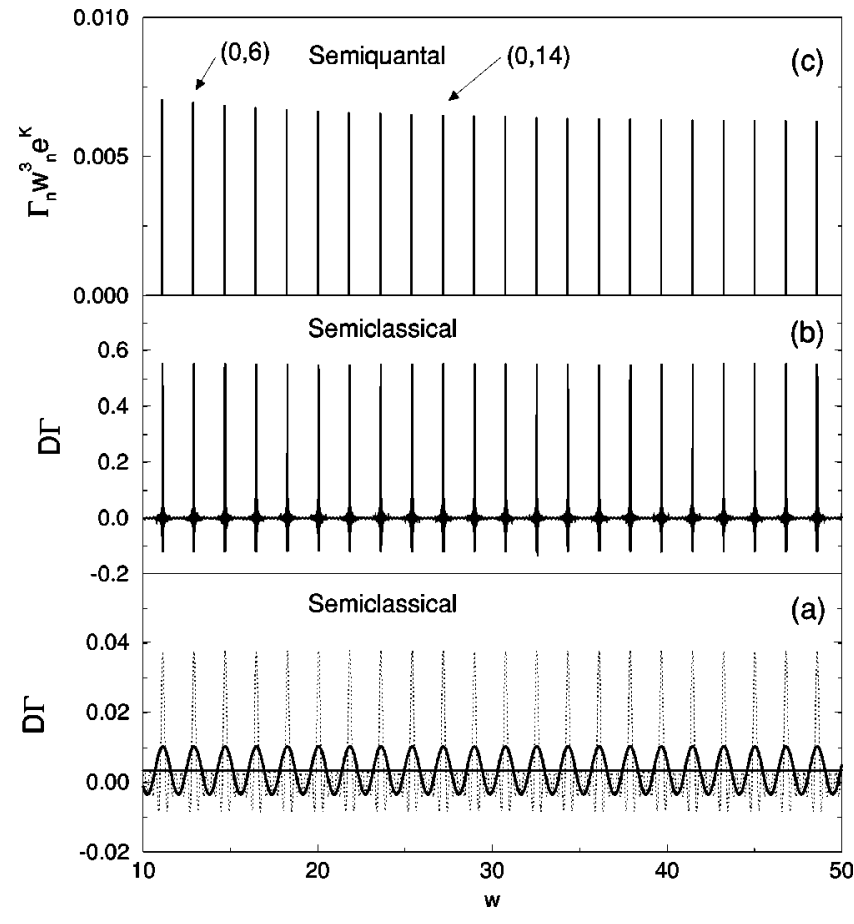

FIG. 6. Comparison between semiclassical (a) and (b) and semiquantal (c) results for the width-weighted spectrum at scaled energy $\varepsilon=-2.1$ (atomic units). In the semiquantal result, some widths are labeled by the quantum numbers $\left(n_{u}, n_{v}\right)$. The straight line in (a) is the background for Eq. (34) (i.e., $M_{v}=0$ ). The solid line with few oscillations is the result from Eq. (34) taking the sum until $\left|M_{v}\right|$ $=1$, and the dotted line is the result of the summation up to $\left|M_{v}\right|$ $=5$. (b) is the result for $\max \left|M_{v}\right|=80$.

Equation (38) is the final result for the semiclassical expression of the width-weighted spectrum (1) for the belowbarrier case in the Stark problem. All quantities are evaluated at $\beta=-1$. The result is a single Fourier series with exponentials that oscillate at multiples of $2 \pi / \hat{J}_{v}$, and with complex amplitudes $D_{M_{v}}$ which depend on the "canonical periods" $\hat{\tau}_{v}, \hat{\tau}_{u}, \hat{\tau}_{K}$ in Eq. (33). Often in the below-saddle case, the quantum correction $\delta$ is negligible.

Figure 6(a) shows the convergence of this sum for $\varepsilon$ $=-2.1$. In this figure we extracted the overall factor $\exp [-w \hat{K}]$. The straight line is the result for $M_{v}=0$. The sine wave is the result of taking two more terms in the sum (i.e., $\left|M_{v}\right|=1$ ) and the dotted line is the result of summation up to $\left|M_{v}\right|=5$. The height of the peaks increases by taking more and more terms, because the Fourier series (38) "converges" to $\delta$ functions. They go to infinity, but their integrated areas are proportional to $\widetilde{\Gamma}_{\mathbf{n}}$. In the truncated sum, the breadths of the peaks in Figs. 6(b) and 6(c) are all nearly equal, so the heights are also proportional to $\widetilde{\Gamma}_{\mathbf{n}}$.

Figures 6(b) and 6(c) compare the semiclassical numerical results from Eq. (38) (with $\left|M_{v}\right|^{\max }=80$ ) with the semiquantal results from Eq. (30). The positions of the maxima for a given state $w_{\mathbf{n}}$ are in perfect agreement and also the overall behavior of the peaks is the same. Of course the Fourier sum (38) gives the expected Gibbs' phenomenon. Otherwise the only discrepancy between semiclassical and semiquantal results is that in the quantum results, $\widetilde{\Gamma}_{\mathbf{n}}$ and the spacing between adjacent $w_{\mathbf{n}}$ 's increase slightly as $w$ decreases, while the semiclassical formula (38) is perfectly periodic in $w$.

In this case our approximations leading to Eq. (38) give an accurate representation of the width-weighted spectrum by describing the periodic family of "downhill states" $\left(n_{u}\right.$ $=0, n_{v}=n-1$ ) [15]. (The decay rates of the "next-todownhill" states are much less than those of the downhill states.) However, the connection between Eq. (38) and the quantized downhill states is not obvious. Equation (38) involves only the properties of the downhill periodic orbit, which has no $u$ motion whatever; in contrast the quantized downhill states have zero-point energy in the $u$ motion and therefore somewhat less energy in the $v$ motion than does the downhill PO. Nevertheless, Eq. (38) gives an accurate representation. In fact, Eq. (38) can be resummed into a set of $\delta$ functions having the right widths and in the right locations. Details are in Appendix B.

Now let us consider the "recurrence spectrum of the width-weighted spectrum', by taking the Fourier transform,

$$
\begin{aligned}
R_{\Gamma}(S)= & \int_{w_{1}}^{w_{2}} e^{-2 i \pi S w}\left[\frac{D \Gamma(w)}{e^{-w \hat{K}}}\right] d w \\
= & \sum_{M_{v}} \frac{-i D_{M_{v}}}{\left(M_{v} \hat{J}_{v}-2 \pi S\right)} \\
& \times\left(e^{i w_{2}\left[M_{v} \hat{J}_{v}-2 \pi S\right]}-e^{i w_{1}\left[M_{v} \hat{J}_{v}-2 \pi S\right]}\right) .
\end{aligned}
$$

Observe that for the Fourier transform we extracted from Eq. (38) the smooth factor $\exp [-w \hat{K}]$. We expect to find peaks located close to values of scaled action $S_{M_{v}}=M_{v} \hat{J}_{v} /(2 \pi)$. At these values the Fourier transform is

$$
\left|R_{\Gamma}\left(S=M_{v} \hat{J}_{v} / 2 \pi\right)\right|^{2} \simeq 4\left|D_{M_{v}}\right|^{2}\left[\frac{w_{1}+w_{2}}{2}\right]^{2},
$$

where

$$
\left|D_{M_{v}}\right|^{2} \simeq \frac{1}{4 \pi^{2}} e^{-2 \pi \hat{\tau}_{K} / \hat{\tau}_{u}}
$$

which is independent of $M_{v}$. This is what we see in Figs. 7(a) and 7(b), where the absolute squares of the Fourier transforms for the semiclassical and semiquantal formulas are plotted as a function of the variable $S$. The range of $w$ used for the Fourier transform is from $w_{1}=10$ to $w_{2}=50$. For this scaled energy, the value of the scaled action calculated at $\beta=-1$ is $\hat{J}_{v} /(2 \pi) \simeq 0.56059$.

Equation (43) was calculated using the approximation given in Appendix A for the denominator of $D_{M_{v}}$ of Eq. (39).

The physical meaning of the exponential factor in Eq. (43) is the following. We note that all of the equations in this section [Eqs. (37)-(43)] involve properties of the downhill orbit (for energies below the saddle, each quantity with a caret is evaluated at $\beta=-1$ ). However, the quantized reso- 


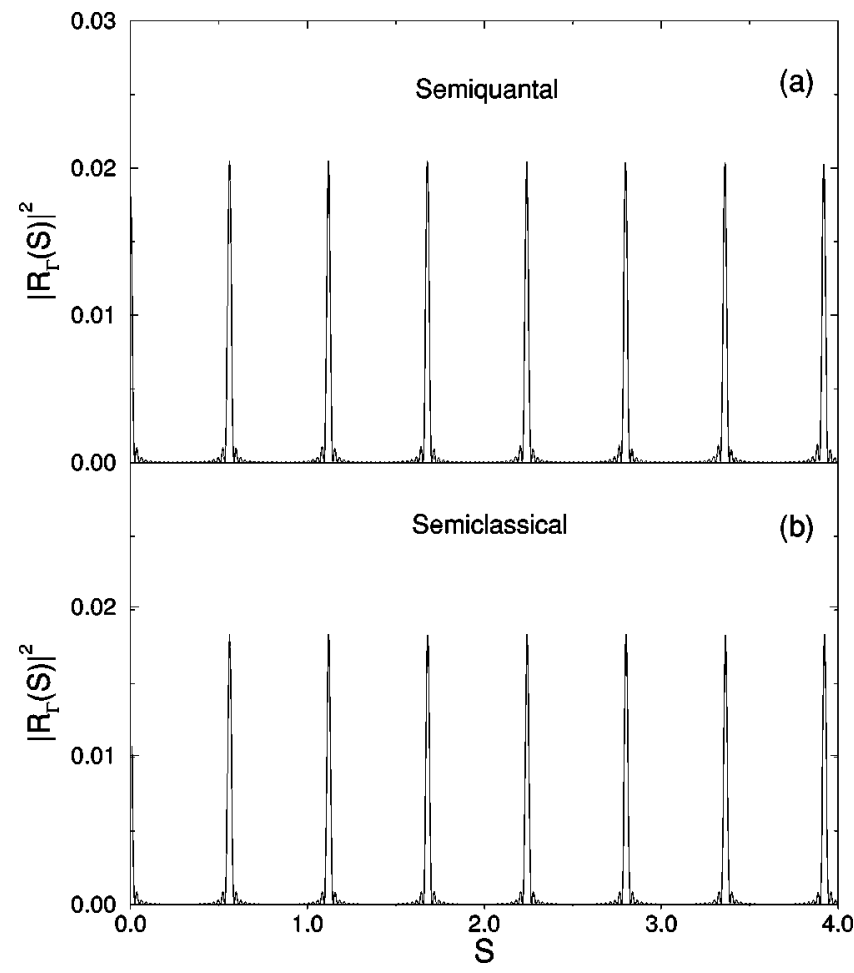

FIG. 7. Comparison between (a) semiclassical and (b) semiquantal results for the absolute square of the Fourier transform in atomic units at the scaled energy $\varepsilon=-2.1$. Peaks in the semiclassical result are located at scaled actions $S_{M_{v}}$ and the highs are proportional to $\left|D_{M_{v}}\right|^{2}$ which is independent of $M_{v}$.

nances have slightly different values of $\beta$-there must be at least the zero-point motion in the $u$ coordinate. Therefore the decay rate must be governed by the tunneling integral $\widetilde{K}(\varepsilon, \beta)$ evaluated at the quantized $\beta_{\mathbf{n}}$, not at $\beta=-1$. We show in Appendix B that the exponential factor in Eq. (43) is the correction that is needed to adjust $\hat{K}$ in Eq. (40) to $\widetilde{K}\left(\varepsilon, \beta_{\mathbf{n}}\right)$.

\section{Above saddle}

The semiclassical formula for the scaled width-weighted spectrum for the above barrier case is also given by Eq. (34). However, the quantities are now calculated at the end point $\beta_{c}$, and the formula involves properties of the "extremal torus," or "last surviving torus" at each scaled energy. As explained earlier, the motion is oscillatory in $v$ if $\beta$ $>\beta_{c}(\varepsilon)$. When $\beta=\beta_{c}(\varepsilon)$, the $v$ motion ascends the dynamical barrier and hangs at the top while $u$ oscillates periodically, and we call the associated torus the extremal torus. For $\beta<\beta_{c}(\varepsilon)$, the particle goes over the dynamical barrier and escapes (see also Fig. 2). For $\beta$ close to $\beta_{c}(\varepsilon)(-2$ $\lesssim \varepsilon \lesssim 0$ ), the parabolic phase correction $\delta$ cannot be neglected. Classically, if the electron stops at the top of the barrier, the corresponding period goes to infinity. Therefore derivatives of $\widetilde{J}_{v}(\varepsilon, \beta)$ diverge as $\beta \rightarrow \beta_{c}(\varepsilon)$. In the quantum case, however, the uncertainty principle prevents such localization of the particle, and the period for reflection (or time delay for transmission) at the top of the barrier is finite. Including $\delta$ in

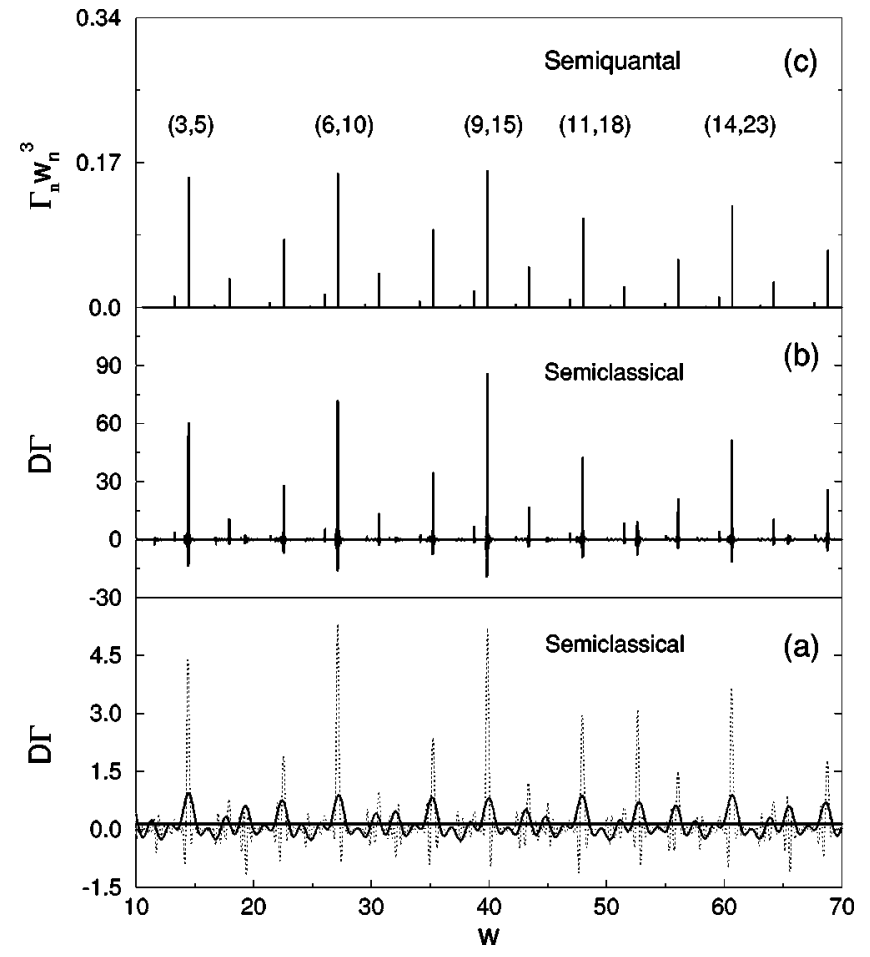

FIG. 8. Comparison between semiclassical (a) and (b), and semiquantal (c) results for the width-weighted spectrum at scaled energy $\varepsilon=-1.5$ (atomic units). The straight line in (a) is the result from Eq. (34) for $M_{u}=M_{v}=0$. The solid line with few oscillations is the result from Eq. (34) taking the sum until $\left|M_{u}\right|=\left|M_{v}\right|=1$, and the dotted line is the result from the summation until $\left|M_{u}\right|=\left|M_{v}\right|$ $=5$. (b) is the result for $\left|M_{u}\right|^{\max }=300,\left|M_{v}\right|^{\max }=200$. In the semiquantal result, some widths are labeled by the quantum numbers $\left(n_{u}, n_{v}\right)$.

Eq. (33), the divergence of the derivative of $\widetilde{J}_{v}(\varepsilon, \beta)$ is canceled by a corresponding divergence in the derivative of $\delta$, so $\hat{\tau}_{v}$ is finite.

In Fig. 8(c) is shown the semiquantal width-weighted spectrum for $\varepsilon=-1.5$ together with the semiclassical results, again displaying the convergence properties. The straight line in Fig. 8(a) is the "smooth background," i.e., the result for $M_{u}=M_{v}=0$. The solid line with few oscillations is the result of taking eight more terms in the sum (i.e., up to $\left|M_{u}\right|=\left|M_{v}\right|=1$ ) and the dotted line is for summation up to $\left|M_{u}\right|=\left|M_{v}\right|=5$. Figure $8(\mathrm{~b})$ shows the sum up to $\left|M_{u}\right|^{\max }=80,\left|M_{v}\right|^{\max }=60$. The positions of the maxima are in good agreement with the semiquantal result, and also the overall behavior of the peaks is the same.

For the recurrence spectrum we expect to find peaks located at values of scaled action $S_{M_{u}, M_{v}}=\left(M_{u} \hat{J}_{u}\right.$ $\left.+M_{v} \hat{J}_{v}\right) /(2 \pi)$. In Figs. 9(a) and 9(b), the (normalized) absolute square of the Fourier transform is plotted as a function of the variable $S$. For this scaled energy, the value of the scaled actions of the extremal torus are $\hat{J}_{u} /(2 \pi) \simeq 0.23796$ and $\hat{J}_{v} /(2 \pi) \simeq 0.38985$. The range of $w$ used for the Fourier transform is from $w_{1}=10$ to $w_{2}=120$. The needles under the peaks correspond to the values of 


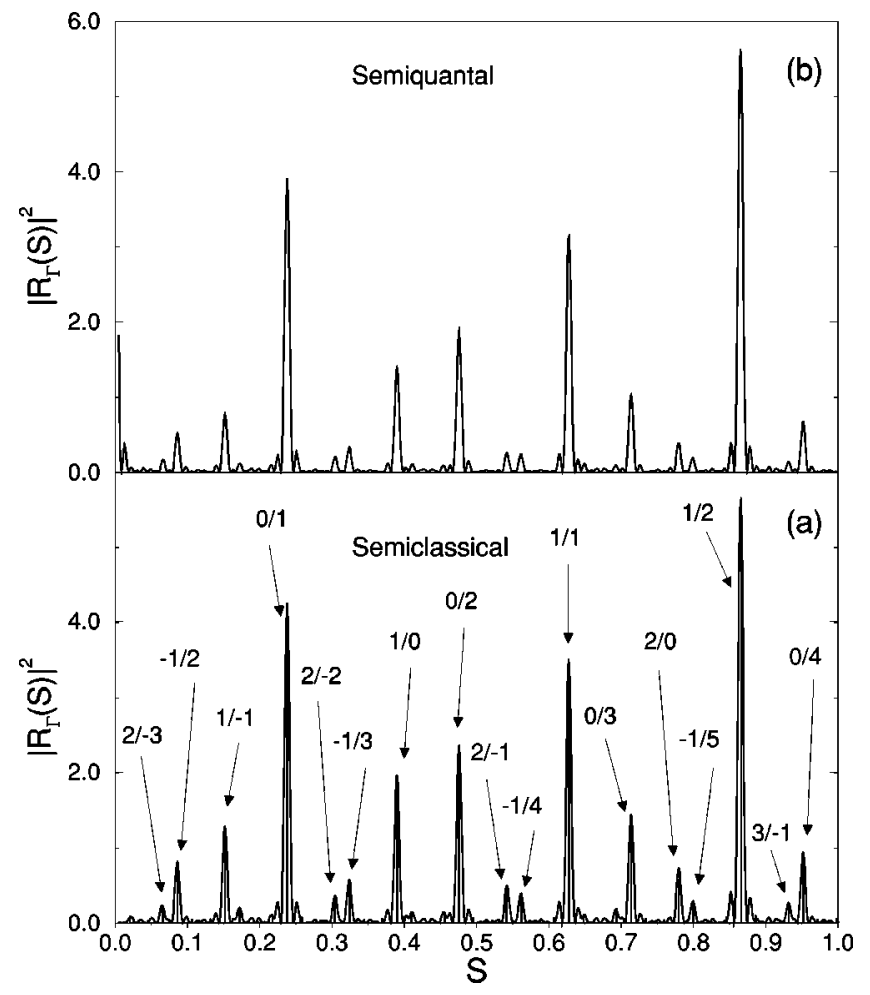

FIG. 9. Comparison between (a) semiclassical and (b) semiquantal results for the absolute square of the Fourier transform (in atomic units) at the scaled energy $\varepsilon=-1.5$. Peaks in the semiclassical result are located at scaled actions $S_{M_{u}, M_{v}}$ and they are labelled by the relation $\left(M_{v} / M_{u}\right)$. The needles under these peaks correspond to the values of $\left|D_{M_{u}, M_{v}}\right|^{2}$.

$$
\left|D_{M_{u}, M_{v}}\right|^{2}=\frac{\hat{\tau}_{u}^{2}}{16 \pi^{4}\left[\hat{\tau}_{K}^{2}+\left(M_{u} \hat{\tau}_{u}-M_{v} \hat{\tau}_{v}\right)^{2}\right]} .
$$

In this case the relative heights of the peaks in the Fourier transform depend of course on the values of $M_{u} / M_{v}$. Here, the importance of the parabolic correction $\delta$ is manifested, because without it, $\hat{\tau}_{v}$ would be infinite.

Each peak in the Fourier transform [see Fig. 9(a)] can be indentified with a given loop on the extremal torus, having $M_{u} u$ cycles and $M_{v} v$ cycles. In general these loops do not correspond to closed orbits or to periodic orbits. For example, we see a large $1 / 1$ peak, but the frequencies of the $u$ and $v$ motions are never equal, and no (1/1) periodic or closed orbit exists. In addition, we can observe peaks with negative cycle numbers, such as $M_{v} / M_{u}=-1 / 2$. These could be regarded as the contribution of paths on the torus integrated "backward in time" in one coordinate $\left(M_{v}\right.$ $=-1$ ), together with motions integrated "forward in time", in the other coordinate $\left(M_{u}=2\right)$. Clearly these loops do not correspond to classical orbits.

This concludes our analysis of width-weighted spectra in the Stark system. To summarize, we have shown that an appropriate end point approximation (32) applied to the integral representation (31) leads to a formula (35) for the width-weighted spectrum that involves actions and canonical periods of the extremal torus.

\section{OSCILLATOR-STRENGTH DENSITY}

Now let us turn to a more familiar weighted spectrumthe oscillator-strength density. We will show that a stationary-phase approximation applied to the general expression (18) leads to the formulas of closed-orbit theory [2].

The observable quantity - the oscillator strength for the transition from a given initial state $\mathbf{i}$ to a final state $\mathbf{n}-$ is defined as

$$
f_{\mathbf{n}}^{\mathbf{i}}=2\left(E_{\mathbf{n}}-E_{\mathbf{i}}\right)|\langle\mathbf{i}|D| \mathbf{n}\rangle|^{2},
$$

where $D$ is the relevant component of the dipole operator; then according to Eq. (1) the oscillator-strength density $D f(E)$ is

$$
D f(E)=\sum_{\mathbf{n}} f_{\mathbf{n}}^{\mathbf{i}} \delta\left(E-E_{\mathbf{n}}\right)
$$

For a regular system (such as a hydrogen atom in an external electric field), the semiclassical approximation gives the following expression for $f_{\mathbf{n}}^{\mathbf{i}}$ :

$$
f_{\mathbf{n}}^{\mathbf{i}}=4(2 \pi)^{d+1}\left(E_{\mathbf{n}}-E_{\mathbf{i}}\right)\left|\frac{\partial(E, \boldsymbol{\beta})}{\partial(\mathbf{J})}\right|\left|\mathcal{Y}_{\boldsymbol{\beta}}\left(\Omega_{\mathbf{n}}\right)\right|^{2}
$$

The function

$$
\left|\mathcal{Y}_{\boldsymbol{\beta}}(\Omega)\right|^{2}=\left.\left|\frac{\partial(\Omega)}{\partial(\boldsymbol{\beta})}\right| \mathcal{Y}(\Omega)\right|^{2}
$$

is proportional to the angular distribution of the electrons ejected from the atom

$$
|\mathcal{Y}(\Omega)|^{2}=\left(1 / 8 \pi^{2}\right)\left|\left\langle\mathbf{i}|D| \Psi_{\Omega_{(\boldsymbol{\beta})}}^{C}\right\rangle\right|^{2},
$$

$\Psi_{\Omega}^{C}$ is a zero-energy scattering wave function (normalized to the $\delta$ function of energy) with a given value of the integrals of motion $\boldsymbol{\beta}$.

These formulas were explained and derived in Refs. $[10,11]$. They involve the assumption already stated that the trajectories are regular and bounded, and form tori labeled by action variables $\mathbf{J}$ or conserved quantities $(E, \boldsymbol{\beta})$.

The formulas involve an additional assumption. Near the nucleus the trajectories associated with the semiclassical approximation for the excited state $\mathbf{n}$ are similar to those for zero-energy Coulomb scattering. For each torus, there is a central trajectory that comes on a straight line exactly to the nucleus, turns around, and goes back out on itself. Nearby the trajectories form parabolas symmetric about that line. The angular variables $\Omega$ define the spatial orientation of the straight line: in the cylindrically symmetric case $(d=2) \Omega$ is $\cos \theta$ ( $\theta$ being the polar angle), while in the threedimensional case with no cylindrical symmetry, $\Omega$ $=(\cos \theta, \phi)$ represents both polar and azimuthal angles. We assume that there is a smooth invertible relationship between the $d-1$ conserved quantities $\boldsymbol{\beta}$ and the $d-1$ angles represented by $\Omega$. In Eq. (47) $\Omega$ is evaluated at $\Omega_{\mathbf{n}} \equiv \Omega\left(\boldsymbol{\beta}_{\mathbf{n}}\right)$, so we are evaluating the angular distribution of outgoing waves 
in the direction that "feeds" the quantized eigentorus.

On the other hand, the well-established closed-orbit theory [2] asserts (also in the semiclassical approximation) that the oscillator-strength density may be represented as a sum over the contributions of orbits closed at the nucleus,

$$
D f(E)=D f_{0}(E)+\sum_{\begin{array}{c}
\text { closed orbits } \\
\text { their repetitions }
\end{array}} C_{k}(E) \sin \Delta_{k}(E) .
$$

Here $D f(E)$ is the same quantity as in Eq. (46). $D f_{0}(E)$ is the "background absorption"density that would be present if the electron directly escaped from the atom and never returned. $\Delta_{k}(E)$ is equal to the classical action around the closed orbit, plus certain corrections associated with Maslov indices. $C_{k}(E)$ is a quantity called the recurrence amplitude. It depends on the stabitity of the orbit and is also proportional to the angular distribution of the outgoing electrons $|\mathcal{Y}(\theta, \phi)|^{2}$. (More details can be found in Ref. [2].)

There must be a correspondence between Eqs. (50) and (46), since they both represent the same observable quantity. In order to show that, let us return to formula (18) in Sec. II D. Now we will transform this formula under the assumption that the weighting function $W(E, \boldsymbol{\beta})$ is a slowly varying function. This is true for $W(E, \boldsymbol{\beta})$ given by Eq. (47),

$$
W(E, \boldsymbol{\beta})=4(2 \pi)^{d+1}\left(E-E_{\mathbf{i}}\right)\left|\frac{\partial(E, \boldsymbol{\beta})}{\partial(\mathbf{J})}\right|\left|\mathcal{Y}_{\boldsymbol{\beta}}(\Omega)\right|^{2}
$$

In this expression, it is understood that the angles $\Omega$ are functions of $E$ and $\boldsymbol{\beta}$ as explained earlier.

We will integrate over $\boldsymbol{\beta}$, holding $E$ fixed. The integral is rapidly oscillatory, but it may have a stationary phase point if at some value of $\boldsymbol{\beta}$

$$
\mathbf{M} \cdot\left(\frac{\partial \mathbf{J}}{\partial \beta_{k}}\right)_{E}=0, \quad k=1, \ldots, d-1 .
$$

These points correspond to periodic orbits with winding numbers M. To show this we think of action space as a $d$-dimensional Cartesian space; the vectors $\left(\partial \mathbf{J} / \partial \beta_{k}\right)_{E}$ span the $(d-1)$-dimensional energy surface in action space. They are therefore orthogonal to the frequency vector $\boldsymbol{\omega}=\partial \mathbf{H} / \partial \mathbf{J}$, which is the normal to the energy surface. But if the vector $\mathbf{M}$ is also orthogonal to those same $(d-1)$ vectors, it must also be normal to the energy surface, and therefore collinear with the vector $\boldsymbol{\omega}$. Thus if $\mathbf{M} \propto \boldsymbol{\omega}$, then the frequencies are commensurable, and the associated torus is a rational torus, covered by periodic orbits with winding numbers $\mathbf{M}$. If in the energy range of interest such a periodic orbit exists, then the Mth term has a stationary phase point at $\boldsymbol{\beta}=\hat{\boldsymbol{\beta}}$, and the stationary-phase contribution to the integral over $\boldsymbol{\beta}$ becomes

$$
\begin{aligned}
D \mathcal{W}= & \frac{1}{(2 \pi)^{(d+1) / 2}} \sum_{\{\mathbf{M}\}} \exp (-2 \pi i \mathbf{M} \cdot \boldsymbol{\mu}) W(E, \hat{\boldsymbol{\beta}}) \\
& \times \exp \left[i S(E, \hat{\boldsymbol{\beta}})+i(\pi / 4) \operatorname{sgn}\left(\partial^{2} S / \partial \beta_{j} \partial \beta_{k}\right)\right] \\
& \times\left|\frac{\partial(\mathbf{J})}{\partial(E, \hat{\boldsymbol{\beta}})}\right|\left|\operatorname{det}\left(\partial^{2} S / \partial \beta_{j} \partial \beta_{k}\right)\right|^{-1 / 2} \\
& +\frac{1}{(2 \pi)^{d}} \int d \boldsymbol{\beta} W(E, \boldsymbol{\beta})\left|\frac{\partial(\mathbf{J})}{\partial(E, \boldsymbol{\beta})}\right|,
\end{aligned}
$$

where

$$
S(E, \hat{\boldsymbol{\beta}})=\mathbf{M} \cdot \mathbf{J}(E, \hat{\boldsymbol{\beta}})
$$

is the action around the periodic orbit. The symbol $\{\mathbf{M}\}$ indicates that summation is performed only over the periodic orbits. The symbol sgn denotes the difference between the numbers of positive and negative eigenvalues of a matrix. [The integral in Eq. (53) represents the background term with $\mathbf{M}=0$ where the stationary-phase approximation does not apply.] By this means formulas based upon quantization of action variables are connected to formulas from periodic orbit theory.

If we substitute $W(E, \boldsymbol{\beta})$ in the form (51) into the expression (53), we come to a closed-orbit formula

$$
\begin{aligned}
D f(E)= & 4(2 \pi)^{(d+1) / 2}\left(E-E_{\mathbf{i}}\right) \sum_{\{\mathbf{M}\}} \frac{\exp \left[i S(E, \hat{\boldsymbol{\beta}})-2 \pi i \mathbf{M} \cdot \boldsymbol{\mu}+i(\pi / 4) \operatorname{sgn}\left(\partial^{2} S / \partial \beta_{j} \partial \beta_{k}\right)\right]}{\left|\operatorname{det}\left(\partial^{2} S / \partial \beta_{j} \partial \beta_{k}\right)\right|^{1 / 2}} \\
& \times\left|\mathcal{Y}_{\boldsymbol{\beta}}(\hat{\Omega})\right|^{2}+8 \pi\left(E-E_{\mathbf{i}}\right) \int d \boldsymbol{\beta}\left|\mathcal{Y}_{\boldsymbol{\beta}}(\Omega)\right|^{2} .
\end{aligned}
$$

If the sum in Eq. (55) contains a term with vector index $\mathbf{M}$, it also contains the term with the vector index - M. Grouping the terms into such pairs, we come to expression (50) with

$$
D f_{0}(E)=8 \pi\left(E-E_{\mathbf{i}}\right) \int d \boldsymbol{\beta}\left|\mathcal{Y}_{\boldsymbol{\beta}}(\Omega)\right|^{2},
$$

$$
C_{k}(E)=8(2 \pi)^{(d+1) / 2}\left(E-E_{\mathbf{i}}\right) \frac{\left|\mathcal{Y}_{\boldsymbol{\beta}}(\hat{\Omega})\right|^{2}}{\left|\operatorname{det}\left(\partial^{2} S / \partial \beta_{j} \partial \beta_{k}\right)\right|^{1 / 2}},
$$

$$
\Delta_{k}(E)=S(E, \hat{\boldsymbol{\beta}})-2 \pi \mathbf{M} \cdot \boldsymbol{\mu}-(\pi / 4) \operatorname{sgn}\left(\partial^{2} S / \partial \beta_{j} \partial \beta_{k}\right)
$$


Thus, using Eq. (51) and a stationary-phase approximation, we have arrived at an expression with the same structure as the closed-orbit formula of Du and Delos [2]. However, Eq. (56b) is a new expression for the recurrence amplitude, and Eq. (56c) contains a new expression for the Maslov phases. The new expressions apply only to regular systems, whereas those in Ref. [2] apply to regular or chaotic systems, but in some cases, the new formulas may be easier to compute. In Appendix E we show that Eq. (56b) is equivalent to the corresponding formula from Du and Delos when both apply.

Let us consider again the hydrogen atom in an electric field with $m_{l}=0$. The $z$ component of the Laplace-RungeLenz vector can serve as the integral of motion $\beta$. It is simply related with the ejection angle for the classical orbit starting at the nucleus

$$
\beta=\cos \theta
$$

Below the saddle energy $E_{s}=-2 \sqrt{F}$ (Hartree), all orbits that start at the nucleus $(0 \leqslant \theta \leqslant \pi)$ are bounded. That means that the integration over $\beta$ in the semiclassical formula (56) is performed from -1 to 1 . The angular distribution of ejected electrons does not depend on the angle $\phi$ as the system is axially symmetric. Moreover, in virtue of Eq. (57) $\partial \Omega / \partial \beta$ $=1$ and therefore $\left|\mathcal{Y}_{\beta}(\theta)\right|^{2}=|\mathcal{Y}(\theta)|^{2}$.

The closure condition (52) takes the form

$$
M_{1} \frac{\partial J_{u}}{\partial \beta}+M_{2} \frac{\partial J_{v}}{\partial \beta}=0 \text { or } M_{1} \tau_{u}=M_{2} \tau_{v}
$$

taking into account that $\partial J_{u} / \partial \beta=\tau_{u} / 2$ and $\partial J_{v} / \partial \beta$ $=-\tau_{v} / 2$ [cf. Eq. (33)]. The second derivative of action along a closed orbit, which enters Eq. (56), may be represented in the following form:

$$
\frac{\partial^{2} S}{\partial \beta^{2}}=\frac{M_{1} \tau_{u}}{2} \frac{\partial \ln \left(\tau_{u} / \tau_{v}\right)}{\partial \beta}=\frac{M_{2} \tau_{v}}{2} \frac{\partial \ln \left(\tau_{u} / \tau_{v}\right)}{\partial \beta} .
$$

Taking into account the above expressions and using scaled quantities introduced in Sec. III A, we transform the general semiclassical expression (56) into the form

$$
\begin{gathered}
D f_{0}(E)=8 \pi\left(E-E_{\mathbf{i}}\right) \int_{-1}^{1} d \beta|\mathcal{Y}(\theta)|^{2}, \\
C_{k}(E)=32 \pi^{3 / 2}\left(E-E_{\mathbf{i}}\right) \frac{|\mathcal{Y}(\hat{\theta})|^{2}}{\left[M_{1} \tau_{u} \partial \ln \left(\tau_{u} / \tau_{v}\right) / \partial \beta\right]^{1 / 2}}, \\
\Delta_{k}(E)=w \widetilde{S}(E, \hat{\boldsymbol{\beta}})-\pi M_{1}-\pi M_{2}-\pi / 4 .
\end{gathered}
$$

A comment is necessary about the influence of the end points $\beta_{\min }=-1$ and $\beta_{\max }=1$ in the integral (18). Let us consider the contribution of the end point $\beta_{\max }=1$ (or $\theta$ $=0$ ). If this point is isolated from the stationary points, its contribution $D^{+1} f(E)$ has the following form:

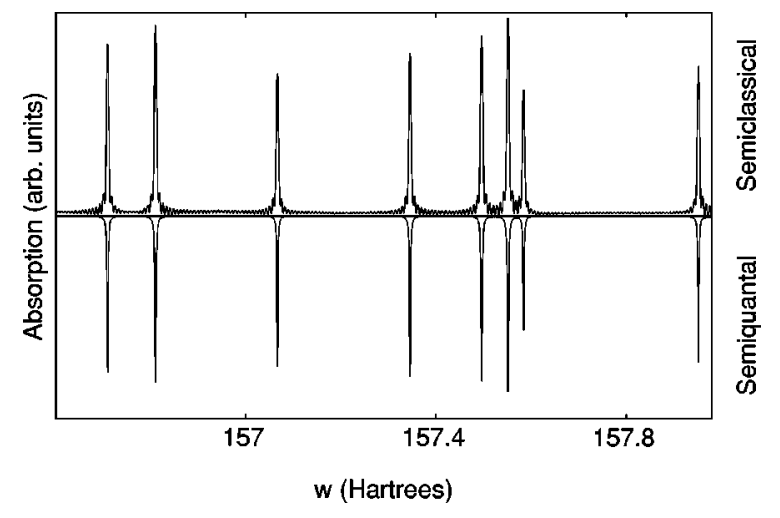

FIG. 10. Scaled Hydrogenic Stark spectrum with scaled energy $\varepsilon \equiv E / \sqrt{F}=-0.4$ plotted as function of scaled variable $w=F^{-1 / 4} . F$ varies around $10 \mathrm{~V} / \mathrm{cm}$, so the energy $E$ varies simultaneously around $4 \mathrm{~cm}^{-1}$ (the principal quantum number is about 100). The result of semiclassical calculation using formulas (50) is drawn as an upper part of this mirror plot. This calculation uses orbits with up to 200 oscillations along each of the parabolic coordinates. The noticeable width of the peaks is due to the truncation of the closedorbit sum. The lower part of the plot is semiquantal calculation according to our formulas (46) and (47). The semiquantal oscillatorstrength density is artificially broadened to match the width of the upper part.

$$
\begin{aligned}
D^{+1} f(E)= & 4\left(E-E_{\mathbf{i}}\right) \sum_{\mathbf{M} \neq \mathbf{0}} \frac{(-1)^{M_{2}} \exp \left[i M_{1}\left(J_{u}-\pi\right)\right]}{i\left(M_{1} \tau_{u}-M_{2} \tau_{v}\right)} \\
& \times|\mathcal{Y}(\theta=0)|^{2} .
\end{aligned}
$$

The formula (36) allows summation over $M_{2}$. We get

$$
D^{+1} f(E)=\frac{8\left(E-E_{\mathbf{i}}\right)}{\tau_{u}}|\mathcal{Y}(\theta=0)|^{2} \sum_{M_{1}=1}^{\infty} \frac{\sin \left[M_{1}\left(J_{u}-\pi\right)\right]}{\sin \left(\pi M_{1} \tau_{u} / \tau_{v}\right)} .
$$

The action variable $J_{u}$ and the periods in this expression are to be calculated at $\beta=1$. [The expression for $D^{-1} f(E)$ can be obtained from Eq. (62) by the interchange of indices $u$ and $v$ and by inverting the total sign. The angular distribution function $|\widetilde{\mathcal{Y}}(\theta)|^{2}$ must be taken at $\theta=\pi$.]

These expressions describe the contributions of two stable orbits of the system: the uphill orbit with ejection angle $\theta$ $=0$ and the downhill orbit with $\theta=\pi$. As was mentioned before, the contributions $D^{ \pm 1} f(E)$ are often small except near a bifurcation [16]. In this case, at some energy, $\tau_{u} / \tau_{v}$ becomes rational (a new closed orbit appears) and some member of the sum (62) goes to infinity. From the mathematical point of view, this is the case of a coalescence of a stationary phase point with the endpoint. The ways of handling this case are well known $[17,16,18]$.

The formulas (60), combined with expressions for the actions, periods, and their derivatives through elliptic integrals reported earlier [19] provides us with an efficient way to calculate the recurrence strengths $\left|C_{k}(E)\right|^{2}$ and the absorption spectrum itself according to Eq. (50). Figure 10 shows an example of the calculation of a Stark absorption spectrum as a sum of the contributions of closed orbits (50). It is 
compared with the semiquantal oscillator-strength density according to the formulas (46) and (47). (In their turn, these formulas are in excellent agreement with numerical calculations using the quantum formula (45), as reported in [11].) In Fig. 10 the semiquantal oscillator-strength density is artificially broadened, and the truncated closed-orbit sum also gives broadened peaks with width inversely proportional to the time of flight of the longest orbits involved in the calculation. The result shown in Fig. 10 requires accounting for orbits making up to 200 oscillations along each of the two parabolic coordinates. That does not represent any computational problem if we use the analytical expressions (60) [20].

\section{CONCLUSION}

To conclude, a semiclassical representation for a regular spectrum weighted by some arbitrary function $W$ was derived. This representation was written as a function of the action variables, and it includes contributions of periodic or closed orbits as well as loops on the extremal torus for systems with tunneling. For photoabsorption, the formulas link the semiquantal theory of photoabsorption [10,11] with closed-orbit theory [2]. As examples, we considered tunneling and photoabsorption in the Stark system.

\section{ACKNOWLEDGMENTS}

This research was supported by NSF. M.W.B. thanks CAPES for financial support.

\section{APPENDIX A}

In this appendix we show how the semiclassical formula [Eqs. (34) and (35)] should be generalized to give correct widths up to the top of the potential barrier. This generalization reveals the role of classical underbarrier motion in determinating the widths.

The uniform expression for the width near the top is similar to Eq. (30),

$$
\Gamma_{\mathbf{n}}=\frac{\frac{\partial J_{u}}{\partial \beta}}{\frac{\partial\left(J_{u}, J_{v}\right)}{\partial(E, \beta)}} \ln \left(1+e^{-K}\right) .
$$

This expression works well even above the top, with proper definition of $J_{v}$. However, we restrict ourselves to the region below the top, where $e^{-K}<1$.

At the top of the barrier the values of the width given by the two formulas, (30) and (A1), differ noticeably. Indeed, formula (30) is proportional to $e^{-K}=1$, whereas the formula (A1) is proportional to $\ln \left(1+e^{-K}\right)=\ln 2 \approx 0.69$ at the top.

In order to employ the semiclassical approximation described in Sec. III C to the uniform expression, let us expand the logarithm in the rhs of Eq. (A1),

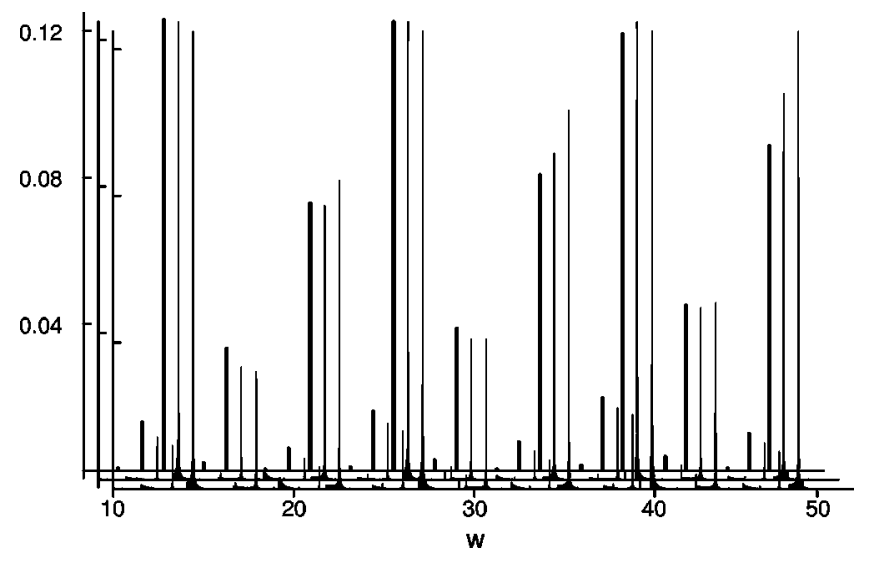

FIG. 11. The uniform semiquantal [Eq. (A1)] spectrum of widths is plotted for the scaled energy $\varepsilon=-1.5$ as bold sticks with height equal to $\Gamma_{\mathbf{n}} w^{3}$ and position $w_{\mathbf{n}}$ satisfying the equality $E_{\mathbf{n}} w_{\mathbf{n}}^{2}=\varepsilon$ (atomic units). The results of summation of the general semiclassical formula (A3)-(A4) are shown as thin lines shifted slightly to the right; the summation includes terms up to $\left|M_{u}\right|$ $=200,\left|M_{v}\right|=200, M_{K}=20$, and only the positive part of the semiclassical graph is shown (cf. Fig. 8). Results of summation of the restricted semiclassical formula (34)-(35) are shown as thin lines shifted farther to the right. The restricted formula (34)-(35) is equivalent to truncating (A3) $-(\mathrm{A} 4)$ to the terms with $M_{K}=1$.

$$
\Gamma_{\mathbf{n}}=-\sum_{M_{K}=1}^{\infty} \frac{\frac{\partial J_{u}}{\partial \beta}}{\frac{\partial\left(J_{u}, J_{v}\right)}{\partial(E, \beta)}}(-1)^{M_{K}} \frac{e^{-M_{K} K}}{M_{K}} .
$$

Now we can repeat all the reasoning of Sec. III C with respect to each member of this series and come to the generalized formula

$$
D \Gamma=\sum_{M_{u}, M_{v}=-\infty}^{\infty} \sum_{M_{K}=1}^{\infty} D_{M_{u}, M_{v}, M_{K}} e^{-w M_{K} \hat{K}+i w\left(M_{u} \hat{J}_{u}+M_{v} \hat{J}_{v}\right)},
$$

where

$$
D_{M_{u}, M_{v}, M_{K}}=-\frac{1}{4 \pi^{2} M_{K}} \frac{(-1)^{M_{u}+M_{v}+M_{K}} \hat{\tau}_{u}}{\left[M_{K} \hat{\tau}_{K}-i\left(M_{u} \hat{\tau}_{u}-M_{v} \hat{\tau}_{v}\right)\right]} .
$$

The terms with $M_{K}=1$ of this triple Fourier sum coincide with expressions (34) and (35). In the other terms, the action $K$ and period of underbarrier motion $\hat{\tau}_{K}$ are multiplied by $M_{K}$. We may interpret these terms as representing multiple underbarrier "oscillations" with pure-imaginary action $i \hat{K}$ and period $i \hat{\tau}_{K}$. The expressions (A3) and (A4) suggest that, to describe the widths near the barrier top, we need to consider a complex extension of the extremal torus. This extension includes "oscillatory motion" or multiple traverses of the classically forbidden region with imaginary momentum. Similar contributions to the spectrum of energy splitting in a double well are discussed by Creigh and Whelan [3].

Figure 11 shows how accounting for multiple oscillations 
under the barrier brings the large semiclassical widths closer to the semiquantal ones (displayed as the bold sticks).

A summation over $M_{K}$ in the generalized expression (A3) can be done exactly above the saddle, when $\hat{K}=0$ on the extremal torus. This way we may get a "renormalized" amplitude $D_{M_{u}, M_{v}}$ to use in the formula (34) near the barrier top,

$$
\begin{aligned}
\widetilde{D}_{M_{u}, M_{v}}= & (-1)^{M_{u}+M_{v}} \frac{i}{(2 \pi)^{2}} \frac{\hat{\tau}_{u}}{M_{v} \hat{\tau}_{v}-M_{u} \hat{\tau}_{u}}[\psi(a+1) \\
& -\psi(a+0.5)-2 \ln 2]
\end{aligned}
$$

with $a$ denoting

$$
a=i \frac{M_{v} \hat{\tau}_{v}-M_{u} \hat{\tau}_{u}}{2 \hat{\tau}_{K}}
$$

and a standard notation $\psi(z)$ for the logarithmic derivative of the gamma function $\Gamma(z), \psi(z)=[\ln \Gamma(z)]^{\prime}$.

\section{APPENDIX B}

Let us start by looking only at the denominator of $D_{M_{v}}$ of Eq. (39)

$$
\begin{aligned}
\sin \left(\pi M_{v} \hat{\tau}_{v} / \hat{\tau}_{u}-i \pi \hat{\tau}_{K} / \hat{\tau}_{u}\right)= & \frac{1}{2 i}\left[e^{i\left(-i \pi \hat{\tau}_{K} / \hat{\tau}_{u}+M_{v} \pi \hat{\tau}_{v} / \hat{\tau}_{u}\right)}\right. \\
& \left.-e^{-i\left(-i \pi \hat{\tau}_{K} / \hat{\tau}_{u}+M_{v} \pi \hat{\tau}_{v} / \hat{\tau}_{u}\right)}\right] \\
\simeq & \frac{1}{2 i} e^{\pi \hat{\tau}_{K} / \hat{\tau}_{u}} e^{i M_{v} \pi \hat{\tau}_{v} / \hat{\tau}_{u}} .
\end{aligned}
$$

We suppose here that the first term on the right side dominates (i.e., $e^{-\pi \hat{\tau}_{K} / \hat{\tau}_{u \ll e} \pi \hat{\tau}_{K} / \hat{\tau}_{u}}$ ). Using this relation in Eq. (38) we have

$$
D \Gamma \simeq \frac{1}{2 \pi} \sum_{M_{v}}(-)^{M_{v}} e^{-\left(w \hat{K}+\pi \hat{\tau}_{K} / \hat{\tau}_{u}\right)} e^{i M_{v}\left(w \hat{J}_{v}-\pi \hat{\tau}_{v} / \hat{\tau}_{u}\right)} .
$$

Now we can interpret $\left(\pi \hat{\tau}_{K} / \hat{\tau}_{u}\right)$ and $\left(\pi \hat{\tau}_{v} / \hat{\tau}_{u}\right)$ as terms which "adjust'" the values of $K$ and $\hat{J}_{v}$ calculated at $\beta$ $=-1$ to values calculated at the quantized values of $\beta_{\mathbf{n}}$. In other words, we use the expansion

$$
\begin{aligned}
& J_{v}\left(E, \beta_{\mathbf{n}}\right) \simeq \hat{J}_{v}-\frac{\pi}{w} \hat{\tau}_{v} / \hat{\tau}_{u}, \\
& K\left(E, \beta_{\mathbf{n}}\right) \simeq \hat{K}+\frac{\pi}{w} \hat{\tau}_{K} / \hat{\tau}_{u} .
\end{aligned}
$$

Substituting relation (B4) in Eq. (B2) we have

$$
D \Gamma=\frac{1}{2 \pi} e^{-w \hat{K}\left(E, \beta_{\mathbf{n}}\right) / \hbar} \sum_{M_{v}}(-)^{M_{v}} e^{i M_{v} w J_{v}\left(E, \beta_{\mathbf{n}}\right)},
$$

which can be rewritten as

$$
D \Gamma=\frac{1}{2 \pi} e^{-K\left(E, \beta_{\mathbf{n}}\right) / \hbar} \sum_{n_{v}} \delta\left[\frac{J_{v}\left(E, \beta_{\mathbf{n}}\right)}{2 \pi \hbar}-\frac{1}{2}-n_{v}\right]
$$

The argument of the delta function is zero when the EBK quantization condition is satisfied. Therefore, the peaks of Eq. (38) are located at quantized values of actions (or at the quantized values of $w_{\mathbf{n}}$ in the scaled spectrum); the amplitude $D_{M_{v}}$ is responsible for this "adjustment" $w_{\beta_{c}} \rightarrow w_{\beta_{\mathbf{n}}}$ in the scaled spectrum.

\section{APPENDIX C}

In this appendix and in Appendices D and $\mathrm{E}$ we connect the formulas for the semiclassical amplitude given in this paper with the standard formulas of closed-orbit theory. Here we derive a preliminary result $[\mathrm{Eq} .(\mathrm{C} 14 \mathrm{c})]$ related to the geometry of the energy surface in action-angle variables.

The energy surface in action space is given by the equation

$$
H(\mathbf{J})=E,
$$

where, as before, $H$ is the Hamiltonian of the integrable system, $\mathbf{J}$ is the set of action variables, and $E$ is the energy of the system. We consider another set of independent conserved quantities $(E, \boldsymbol{\beta})$, where $\boldsymbol{\beta}$ represents $n-1$ quantites spanning the energy surface. We suppose that there is a one-toone relationship between the actions $\mathbf{J}$ and the integrals of motion $(E, \boldsymbol{\beta})$,

$$
\partial \mathbf{J}(E, \boldsymbol{\beta}) / \partial(E, \boldsymbol{\beta}) \neq 0, \infty .
$$

Differentiation of the identity $H(\mathbf{J}(E, \boldsymbol{\beta}))=E$ with respect to one parameter $\beta_{i}(i=1, \ldots, n-1)$ leads to the identity

$$
\partial \mathbf{J} / \partial \beta_{i} \cdot \boldsymbol{\nu}=0
$$

where $\boldsymbol{\nu}=\partial H / \partial \mathbf{J}$ represents the classical frequencies of the system. Identity (C2) expresses the fact that the vector of frequencies is normal to the energy surface.

If we differentiate the same identity with respect to energy, we come to the formula

$$
\partial \mathbf{J} / \partial E \cdot \boldsymbol{\nu}=1 .
$$

The identities (C1) and (C2) hold on all energy surfaces, so we can continue differentiation with respect to $E$ and $\boldsymbol{\beta}$. The results can be written in a uniform way if we write $x_{1}$ $=E, x_{2}=\beta_{1}, \ldots, x_{n}=\beta_{n-1}$. Then the derivative of (C2) or (C4) with respect to any $x_{k}$ leads to

$$
\frac{\partial^{2} \mathbf{J}}{\partial x_{i} \partial x_{k}} \cdot \boldsymbol{\nu}=-\frac{\partial \mathbf{J}}{\partial x_{i}} \cdot \frac{\partial \boldsymbol{\nu}}{\partial x_{k}} .
$$

The frequency vector $\boldsymbol{\nu}(E, \boldsymbol{\beta})$ varies continuously as a function of $(E, \boldsymbol{\beta})$, and at some values of $(E, \boldsymbol{\beta})$ the frequencies may be commensurable. Let $(E, \boldsymbol{\beta})_{\mathbf{M}}$ be the collection of values of $(E, \boldsymbol{\beta})$ such that

$$
\boldsymbol{\nu}(E, \boldsymbol{\beta})_{\mathbf{M}}=\mathbf{M} / T,
$$


where $\mathbf{M}$ is a vector of integers and $T$ is a free parameter. This collection of points is typically a one-dimensional curve in $(E, \boldsymbol{\beta})$ space: given $\mathbf{M}$, for each $E$ there may be a value of $\boldsymbol{\beta}$ such that $\boldsymbol{\nu}(E, \boldsymbol{\beta})$ satisfies Eq. (C5). These points correspond to a rational torus spanned by periodic orbits with frequency ratios (C5).

Now let us consider the action integral around any torus,

$$
S_{\mathbf{M}}(E, \boldsymbol{\beta})=\mathbf{J}(E, \boldsymbol{\beta}) \cdot \mathbf{M} .
$$

This quantity is defined for every torus, and it involves integration $M_{k}$ times around the $k$ th fundamental loop of the torus. We can again consider second derivatives of $S_{\mathbf{M}}(E, \boldsymbol{\beta})$ with respect to $x_{i}, x_{k}$,

$$
\frac{\partial^{2} S_{\mathbf{M}}(E, \boldsymbol{\beta})}{\partial x_{i} \partial x_{k}}=\frac{\partial^{2} \mathbf{J}(E, \boldsymbol{\beta})}{\partial x_{i} \partial x_{k}} \cdot \mathbf{M} .
$$

Let us evaluate this quantity on the M-rational tori, $(E, \boldsymbol{\beta})=(E, \boldsymbol{\beta})_{\mathbf{M}}$, satisfying Eq. (C6). Substituting Eq. (C5) and then Eq. (C4) into Eq. (C7), we obtain

$$
\left.\frac{\partial^{2} S_{\mathbf{M}}}{\partial x_{i} \partial x_{k}}\right|_{(E, \boldsymbol{\beta})_{\mathbf{M}}}=-\left.T \frac{\partial \mathbf{J}}{\partial x_{i}} \cdot \frac{\partial \boldsymbol{\nu}}{\partial x_{k}}\right|_{(E, \boldsymbol{\beta})_{\mathbf{M}}} .
$$

Now let us consider another collection of variables $y_{1}$ $=t, y_{2}=\beta_{1}, \ldots, y_{n}=\beta_{n-1}$, and let us think about the angle variables

$$
\mathbf{w}(t, E, \boldsymbol{\beta})=\boldsymbol{\nu}(E, \boldsymbol{\beta}) t .
$$

We will need the matrix $\boldsymbol{\Omega}(E, \boldsymbol{\beta})$ having elements $\Omega_{j k}(E, \boldsymbol{\beta})$ defined as

$$
\Omega_{j k}(E, \boldsymbol{\beta})=\partial w_{j} / \partial y_{k},
$$

$$
\begin{aligned}
\boldsymbol{\Omega}(E, \boldsymbol{\beta}) & =\frac{\partial(\mathbf{w})}{\partial(t, \boldsymbol{\beta})} \\
& =\left[\begin{array}{cccc}
\nu_{1} & t \partial \nu_{1} / \partial \beta_{1} & \ldots & t \partial \nu_{1} / \partial \beta_{n-1} \\
\nu_{2} & t \partial \nu_{2} / \partial \beta_{1} & \ldots & t \partial \nu_{2} / \partial \beta_{n-1} \\
\vdots & \vdots & \ddots & \vdots \\
\nu_{n} & t \partial \nu_{n} / \partial \beta_{1} & \ldots & t \partial \nu_{n} / \partial \beta_{n-1}
\end{array}\right] .
\end{aligned}
$$

Let us define also the matrix

$$
\begin{gathered}
\mathbf{Y}(E, \boldsymbol{\beta})=[\partial J(E, \boldsymbol{\beta}) / \partial(E, \boldsymbol{\beta})], \\
\Upsilon_{i j}(E, \boldsymbol{\beta})=\partial J_{i} / \partial x_{j} .
\end{gathered}
$$

We need to calculate the matrix product

$$
\boldsymbol{\Sigma}(E, \boldsymbol{\beta})=\mathbf{Y}^{\dagger}(E, \boldsymbol{\beta}) \boldsymbol{\Omega}(E, \boldsymbol{\beta})
$$

and evaluate it on the M-rational torus.

We find immediately

$$
\Sigma_{11}(E, \boldsymbol{\beta})=1,
$$

$$
\begin{gathered}
\Sigma_{i 1}(E, \boldsymbol{\beta})=0 \quad \text { for } i \neq 1, \\
\Sigma_{i+1, k+1}(E, \boldsymbol{\beta})=t \sum_{j=1}^{n} \frac{\partial J_{j}}{\partial \beta_{i}} \frac{\partial \nu_{j}}{\partial \beta_{k}}, \\
\Sigma_{i+1, k+1}(E, \boldsymbol{\beta})_{\mathbf{M}}=-\left.\frac{\partial^{2} S_{\mathbf{M}}(E, \boldsymbol{\beta})}{\partial \beta_{i} \partial \beta_{k}}\right|_{(E, \boldsymbol{\beta})_{\mathbf{M}}} .
\end{gathered}
$$

It follows that the determinant of the $n \times n$ matrix $\mathbf{\Sigma}$ is equal to (minus) the determinant of an $(n-1) \times(n-1)$ matrix

$$
\operatorname{det} \boldsymbol{\Sigma}||_{(E, \boldsymbol{\beta})_{\mathbf{M}}}=-\left.\operatorname{det} \frac{\partial^{2} S_{\mathbf{M}}}{\partial \beta_{i} \partial \beta_{k}}\right|_{(E, \boldsymbol{\beta})_{\mathbf{M}}},
$$

i.e.,

$$
\left.\operatorname{det}\left\{\left[\frac{\partial(\mathbf{J})}{\partial(E, \boldsymbol{\beta})}\right]^{\dagger} \frac{\partial(\mathbf{w})}{\partial(t, \boldsymbol{\beta})}\right\}\right|_{(E, \boldsymbol{\beta})_{\mathbf{M}}}=-\left.\operatorname{det}\left\{\frac{\partial^{2} S_{\mathbf{M}}}{\partial \boldsymbol{\beta} \partial \boldsymbol{\beta}}\right\}\right|_{(E, \boldsymbol{\beta})_{\mathbf{M}}} .
$$

Equations (C13) hold for all (E, $\boldsymbol{\beta})$, while Eqs. (C14) hold on the values $(E, \boldsymbol{\beta})_{\mathbf{M}}$, i.e., on the M-rational torus. The transpose operation can be omitted because we are evaluating a determinant.

The formulas $(\mathrm{C} 14 \mathrm{c}),(\mathrm{C} 13 \mathrm{a})$ and $(\mathrm{C} 13 \mathrm{~b})$ have a simple geometrical meaning related to the energy surface in action space, $H(\mathbf{J})=E$. At any point on this surface, we can consider the rows of the matrix $\mathbf{Y}^{\dagger}$ as coordinate axes. The axes defined by the vectors $\partial(\mathbf{J}) / \partial \beta_{i}, \quad i=1, \ldots, n-1$ are tangent to the energy surface $H(\mathbf{J})=E$. The axis $\partial(\mathbf{J}) / \partial E$ is normal to the energy surface and hence to all other $n-1$ vectors.

Let us also consider as vectors the columns of the matrix $\boldsymbol{\Omega}(E, \boldsymbol{\beta})=\partial(\mathbf{w}) / \partial(t, \boldsymbol{\beta})$ represented in Eq. $(\mathrm{C} 10 \mathrm{~b})$. The first column is the frequency vector $\boldsymbol{\nu}=\partial H / \partial \mathbf{J}$ and therefore it is also normal to the energy surface.

The coordinates of these vectors in the above-described basis form the elements of the matrix product in the left-hand side (lhs) of Eq. (C14c). As the vector $\boldsymbol{\nu}$ is perpendicular to the energy surface, its dot product with $n-1$ basis vectors $\partial(\mathbf{J}) / \partial \beta_{i}$ is zero. This is the content of Eq. $(\mathrm{C} 13 \mathrm{~b})$, and it reduces the dimensionality of the determinant by one, as expressed in Eq. (C14c).

\section{APPENDIX D}

The above result, Eq. $(\mathrm{C} 14 \mathrm{c})$, is useful for evaluating the amplitude of a semiclassical wave function. We consider a regular system, for which all trajectories form tori. Eigenfunctions correspond to "eigentori," i.e., tori on which the action variables are quantized. We consider a wave function for such a system which is locally a solution to the stationary Schrödinger equation in a given domain; however, we consider a wave function which does not correspond to a single eigenfunction. Two examples of the types of wave functions we consider are the Green function and the wave function associated with closed-orbit theory. These wave functions 
are constructed using Maslov's approximation: start from an $(n-1)$-dimensional surface in the $n$-dimensional configuration space, erect normals to that surface, and let the normal at $\mathbf{q}_{0}$ be the direction of the initial momentum at $\mathbf{q}_{0}$. Integrate Hamilton's equations to construct a momentum-vector field $\mathbf{p}(\mathbf{q})$, and simultaneously integrate to find $S(\mathbf{q})=\int_{\mathbf{q}_{0}}^{\mathbf{q}} \mathbf{p} \cdot d \mathbf{q}$. Then the wave function $\psi(\mathbf{q})$ is related to its value on the initial surface by the formula

$$
\psi(\mathbf{q})=\psi\left(\mathbf{q}_{0}\right)\left|J\left(\mathbf{q}_{0}\right) / J(\mathbf{q})\right|^{1 / 2} \exp i[S(\mathbf{q}) / \hbar-\mu \pi / 2],
$$

where

$$
J(\mathbf{q})=\partial \mathbf{q}(t) / \partial\left(t, \mathbf{q}_{0}\right) .
$$

This Jacobian probes the dependence of a trajectory end point $\mathbf{q}(t)$ on the time of flight $t$ and on the $(n-1)$ coordinates $\mathbf{q}_{0}$ of the starting point on the initial surface [21].

Let us assume that the family of initial conditions $\mathbf{p}_{0}\left(\mathbf{q}_{0}\right)$ does not correspond to a single torus. For each $\mathbf{q}_{0}$ on the initial surface there is a $\mathbf{p}_{0}$, and the pair $\left(\mathbf{p}_{0}, \mathbf{q}_{0}\right)$ correspond to initial conditions for a torus having energy $E$ and other conserved quantities $\boldsymbol{\beta}$. All tori have the same energy, but each has its own value of $\boldsymbol{\beta}$-i.e., $\boldsymbol{\beta}$ is a function of $\mathbf{q}_{0}$. The family of orbits evolves from the initial surface, and each orbit lies on a distinct torus.

Embedded in the family of tori are rational tori, corresponding to periodic orbits. Let us suppose that some $\mathbf{q}_{0}$ $=\mathbf{q}_{0}^{\mathbf{M}}$ on the initial surface corresponds to $(E, \boldsymbol{\beta})_{\mathbf{M}}$, the M-rational torus. The orbit emanating from this point is the M-periodic orbit, and its action integral is equal to Eq. (C6), and therefore it obeys Eqs. (C8) and $(\mathrm{C} 14 \mathrm{c})$. We derive here a formula [Eq. (D10)] for the Jacobi determinant as the orbit returns to the initial point. For convenience, we take this point to be the origin of coordinates: $\mathbf{q}=\mathbf{0}$ on the initial surface at $\mathbf{q}_{0}^{\mathbf{M}}$.

For a regular system, we can describe motion in conventional coordinates and momenta $(\mathbf{q}, \mathbf{p})$ or in action-angle coordinates $(\mathbf{w}, \mathbf{J})$ where the actions $\mathbf{J}$ are conserved and cordinates $\mathbf{w}$ vary modulo 1 for bound motion. We can define the w's such that for all $\mathbf{J}, \mathbf{w}=\mathbf{0}$ corresponds to $\mathbf{q}=\mathbf{0}$.

In general, $\mathbf{q}=\mathbf{q}(\mathbf{w}, \mathbf{J})$ and we can represent the Jacobi matrix $\partial(\mathbf{q}) / \partial\left(t, \mathbf{q}_{0}\right)$ as

$$
\frac{\partial(\mathbf{q})}{\partial\left(t, \mathbf{q}_{0}\right)}=\left(\frac{\partial(\mathbf{q})}{\partial(\mathbf{w})}\right) \frac{\partial(\mathbf{w})}{\mathbf{J}}+\left(\frac{\partial(\mathbf{q})}{\partial\left(t, \mathbf{q}_{0}\right)}\right) \frac{\partial(\mathbf{J})}{\partial\left(t, \mathbf{q}_{0}\right)} .
$$

When the periodic orbit completes its cycle, $\mathbf{w}=\mathbf{M}$ and $\mathbf{q}=\mathbf{0}$, so $\partial(\mathbf{q}) /\left.\partial(\mathbf{J})\right|_{\mathbf{w}=\mathbf{M}}=\mathbf{0}$ and, according to Eqs. (D2) and (D3), we have at closure

$$
J_{t}\left(\mathbf{q}, \mathbf{q}_{0}\right)=\left(\frac{\partial(\mathbf{q})}{\partial(\mathbf{w})}\right) \frac{\partial(\mathbf{w})}{\mathbf{J}} .
$$

We assumed already that at each energy $E$, there is a one-to-one correspondence between points on the initial surface $\mathbf{q}_{0}$ and values of the conserved quantities $\boldsymbol{\beta}$. This allows us to write

$$
\frac{\partial(\mathbf{w})}{\partial\left(t, \mathbf{q}_{0}\right)}=\frac{\partial(\mathbf{w})}{\partial(t, \boldsymbol{\beta})} \frac{\partial(t, \boldsymbol{\beta})}{\partial\left(t, \mathbf{q}_{0}\right)},
$$

where the last matrix has the form

$$
\frac{\partial(t, \boldsymbol{\beta})}{\partial\left(t, \mathbf{q}_{0}\right)}=\left[\begin{array}{cc}
1 & \mathbf{0} \\
\mathbf{0} & \partial(\boldsymbol{\beta}) / \partial\left(\mathbf{q}_{0}\right)
\end{array}\right] .
$$

Now we will make several transformations involving results of Appendix C,

$$
\begin{aligned}
J_{t}\left(\mathbf{q}, \mathbf{q}_{0}\right) & =\frac{\partial(\mathbf{q})}{\partial(\mathbf{w})} \frac{\partial(\mathbf{w})}{\partial(t, \boldsymbol{\beta})} \frac{\partial(t, \boldsymbol{\beta})}{\partial\left(t, \mathbf{q}_{0}\right)} \\
& =\frac{\partial(\mathbf{q})}{\partial(\mathbf{w})} \mathbf{\Omega} \frac{\partial(t, \boldsymbol{\beta})}{\partial\left(t, \mathbf{q}_{0}\right)} \\
& =\frac{\partial(\mathbf{q})}{\partial(\mathbf{w})} \mathbf{Y}^{\dagger-1} \mathbf{Y}^{\dagger} \boldsymbol{\Omega} \frac{\partial(t, \boldsymbol{\beta})}{\partial\left(t, \mathbf{q}_{0}\right)} \\
& =\frac{\partial(\mathbf{q})}{\partial(\mathbf{w})} \mathbf{Y}^{\dagger-1} \mathbf{\Sigma} \frac{\partial(t, \boldsymbol{\beta})}{\partial\left(t, \mathbf{q}_{0}\right)} \\
& =\frac{\partial(\mathbf{q})}{\partial(\mathbf{w})}\left[\frac{\partial(E, \boldsymbol{\beta})}{\partial(\mathbf{J})}\right]^{\dagger} \mathbf{\Sigma} \frac{\partial(t, \boldsymbol{\beta})}{\partial\left(t, \mathbf{q}_{0}\right)} \\
& \rightarrow \frac{\partial(\mathbf{q})}{\partial(\mathbf{w})} \frac{\partial(E, \boldsymbol{\beta})}{\partial(\mathbf{J})} \mathbf{\Sigma} \frac{\partial(t, \boldsymbol{\beta})}{\partial\left(t, \mathbf{q}_{0}\right)}
\end{aligned}
$$

Here we have successively substituted Eqs. (C10a), (C11a), and (C12). Equation (D7f) applies because we will take the determinant of the matrix $J_{t}$, so the adjoint does not matter.

Finally, we use the fact that the transformation $(\mathbf{q}, \mathbf{p}) \leftrightarrow(\mathbf{w}, \mathbf{J})$ is canonical, and therefore has a generating function $S(\mathbf{q}, \mathbf{J})$ such that

$$
\mathbf{p}(\mathbf{q}, \mathbf{J})=\partial S(\mathbf{q}, \mathbf{J}) / \partial \mathbf{q} .
$$

We can express this as a function of $(E, \boldsymbol{\beta})$, writing

$$
\begin{gathered}
S(\mathbf{q} ; E, \boldsymbol{\beta}) \equiv S(\mathbf{q}, \mathbf{J}(E, \boldsymbol{\beta})), \\
\mathbf{p}(\mathbf{q} ; E, \boldsymbol{\beta})=\partial S(\mathbf{q} ; E, \boldsymbol{\beta}) / \partial \mathbf{q} .
\end{gathered}
$$

Let us use this representation to rewrite the first two factors in Eq. (D7f)

$$
\begin{aligned}
\frac{\partial(\mathbf{q})}{\partial(\mathbf{w})} \frac{\partial(E, \boldsymbol{\beta})}{\partial(\mathbf{J})} & \rightarrow \frac{\partial(\mathbf{q}, E, \boldsymbol{\beta})}{\partial(\mathbf{w}, \mathbf{J})} \\
& =\frac{\partial(\mathbf{q}, \mathbf{p})}{\partial(\mathbf{w}, \mathbf{J})} \frac{\partial(\mathbf{q}, E, \boldsymbol{\beta})}{\partial(\mathbf{q}, \mathbf{p})} \\
& =\frac{\partial(E, \boldsymbol{\beta})}{\partial(\mathbf{p})}
\end{aligned}
$$




$$
=\left[\frac{\partial^{2} S}{\partial(\mathbf{q}) \partial(E, \boldsymbol{\beta})}\right]^{-1} .
$$

Again these hold because we will take determinants. Equation (D9a) holds because the transformation $\mathbf{J} \leftrightarrow(E, \boldsymbol{\beta})$ is independent of $\mathbf{w}$; Eq. (D9b) involves the function $\mathbf{p}(\mathbf{q} ; E, \boldsymbol{\beta})$ $=\mathbf{p}(\mathbf{q}, \mathbf{J})$; Eq. (D9c) holds because the transformation $(\mathbf{q}, \mathbf{p}) \leftrightarrow(\mathbf{w}, \mathbf{J})$ preserves volume, and the rest is obvious.

Combining all the above and Eq. (C14b), we find

$$
\operatorname{det}\left[J_{t}\left(\mathbf{q}, \mathbf{q}_{0}\right)\right]=-\frac{\operatorname{det}\left[\frac{\partial^{2} S_{\mathbf{M}}}{\partial \boldsymbol{\beta} \partial \boldsymbol{\beta}}\right] \operatorname{det}\left[\frac{\partial(\boldsymbol{\beta})}{\partial\left(\mathbf{q}_{0}\right)}\right]}{\operatorname{det}\left[\frac{\partial^{2} S}{\partial(\mathbf{q}) \partial(E, \boldsymbol{\beta})}\right]} .
$$

This formula is general: it does not involve any of the particular assumptions of closed-orbit theory. We used only the following assumptions: (a) the system is regular, so each orbit lies on a torus characterized by conserved quantities $(E, \boldsymbol{\beta})$; (b) each orbit starts from a surface spanned by variables $\mathbf{q}_{0}$ and there is a one-to-one correspondence between conserved quantities $\boldsymbol{\beta}$ and $\mathbf{q}_{0} ;$ (c) one of these trajectories lies on the M-rational torus, and so it is a periodic orbit with frequency ratio $\mathbf{M}$; (d) we evaluate the classical density Jacobian $J_{t}\left(\mathbf{q}, \mathbf{q}_{0}\right)$ at the final time on that periodic orbit. This formula therefore might be widely useful in various applications of periodic-orbit theory.

\section{APPENDIX E}

Finally we compare two semiclassical expressions for the recurrence amplitude: Eq. (60) that holds for a regular system with any number of degrees of freedom, and the result of closed-orbit theory by Du and Delos [2], Eq. (5.13a),

$$
C=\left(E-E_{i}\right) 2^{19 / 4} \pi^{3 / 2} r_{0}^{-1 / 4}\left(\sin \theta_{i} \sin \theta_{f}\right){ }^{1 / 2} A_{2}\left|\mathcal{Y}\left(\theta_{i}\right) \mathcal{Y}^{*}\left(\theta_{f}\right)\right|
$$

(in Hartrees; all notation will be defined below). This formula (E1) is valid for axially symmetric systems with a regular or chaotic spectrum. These two formulas must give the same values in the common area of their applicability-for axially-symmetric regular systems.

We consider the case that the closure time of the orbit is the same as the period of the orbit. (This holds for all orbits for an atom in an electric field, and for some of the orbits for an atom in a magnetic field. In other cases the period is twice the closure time; then the formulas and the analysis are more complicated.) In the case we consider, the closed orbit is a periodic orbit with frequency ratio $\mathbf{M}$, so it lies on the M-rational torus, and all the equations derived in Appendices $\mathrm{C}$ and $\mathrm{D}$ are valid. Furthermore, $\theta_{i}=\theta_{f}$, and Eq. (E1) may be rewritten as

$$
C=\left(E-E_{i}\right) 2^{19 / 4} \pi^{3 / 2} r_{0}^{-1 / 4} \sin \theta_{f} A_{2}\left|\mathcal{Y}\left(\theta_{f}\right)\right|^{2} .
$$

The two-dimensional semiclassical amplitude $A_{2}$ is simply related to the three-dimensional semiclassical amplitude $A_{3}$ ([2], Eq. (3.12b))

$$
\begin{gathered}
A_{3}\left(\mathbf{r}, \mathbf{q}_{0}\right)=A_{2}\left(r, \theta ; r_{0}, \theta_{0}\right) \sqrt{r_{0}^{2} \sin \theta_{0} / r^{2} \sin \theta}, \\
A_{3}\left(\mathbf{q}, \mathbf{q}_{0}\right)=\sqrt{J_{t \rightarrow 0}\left(\mathbf{q}, \mathbf{q}_{0}\right) / J_{t}\left(\mathbf{q}, \mathbf{q}_{0}\right)}, \\
J_{t}\left(\mathbf{q}, \mathbf{q}_{0}\right)=\partial(\mathbf{q}(t)) / \partial\left(t, \mathbf{q}_{0}\right) .
\end{gathered}
$$

In the calculations of atomic photoabsorption, the initial surface is a sphere of small radius $r_{0}$ that lies in the zone where the Coulomb field is dominant.

As the wave leaves the initial surface, the Jacobian $J_{t \rightarrow 0}\left(\mathbf{q}, \mathbf{q}_{0}\right)$ is easily calculated

$$
J_{t}\left(\mathbf{q}, \mathbf{q}_{0}\right)=\frac{\partial(\mathbf{q}(t))}{\partial\left(t, \mathbf{q}_{0}\right)}=\mathbf{v} \cdot\left(\frac{\partial \mathbf{q}}{\partial q_{0,1}} \times \frac{\partial \mathbf{q}}{\partial q_{0,2}}\right)
$$

with $q_{0,1}$ and $q_{0,2}$ being coordinates of the vector $\mathbf{q}_{0}$ parametrizing the initial surface (sphere in our case). As $t \rightarrow 0$, the velocity vector $\mathbf{v}$ is perpendicular to the initial sphere and we deal with an ordinary product of velocity times Jacobian of transition from Cartesian coordinates to parameters $\mathbf{q}_{0}$ on the sphere

$$
J_{t \rightarrow 0}\left(\mathbf{q}, \mathbf{q}_{0}\right)=v \frac{\partial(\mathbf{a})}{\partial\left(\mathbf{q}_{0}\right)} .
$$

In the region of Coulomb field dominance $v \approx[2(E$ $+1 / r)]^{1 / 2} \approx(2 / r)^{1 / 2}$. If $\mathbf{q}_{0}$ are spherical angles, $\mathbf{q}_{0}=(\theta, \phi)$ then we have

$$
J_{t \rightarrow 0}\left(\mathbf{q}, \mathbf{q}_{0}\right)=v r^{2} \sin \theta=(2 / r)^{1 / 2} r^{2} \sin \theta .
$$

Now we need the Jacobian $J_{t}\left(\mathbf{q}, \mathbf{q}_{0}\right)$ when the wave returns to the initial point along a periodic orbit. For this, we just substitute Eq. (D10) in Eq. (E4), and obtain

$$
A_{3}\left(\mathbf{q}, \mathbf{q}_{0}\right)=\left(\frac{2}{r}\right)^{1 / 4}\left|\frac{\operatorname{det} \frac{\partial(\mathbf{a})}{\partial(\boldsymbol{\beta})} \operatorname{det} \frac{\partial^{2} S}{\partial(E, \boldsymbol{\beta}) \partial(\mathbf{q})}}{\operatorname{det}\left[\frac{\partial^{2} S}{\partial(\boldsymbol{\beta}) \partial(\boldsymbol{\beta})}\right]}\right|^{1 / 2} .
$$

Finally we make the standard approximation of closed-orbit theory of atomic spectra: the wave returning to the nucleus is similar to a zero-energy Coulomb scattering wave. The function $S(\mathbf{q} ; E, \boldsymbol{\beta})$ then corresponds to the phase of this returning wave. In Ref. [10], Eq. (4.2), we gave the formula for the cylindrically symmetric case

$$
\begin{aligned}
S\left(r, \theta, E, \theta_{f}\right)= & -\left\{2 r^{1 / 2}+\frac{1}{3} E r^{3 / 2}\left[2-\cos \left(\theta-\theta_{f}\right)\right]\right\} \\
& \times\left[1+\cos \left(\theta-\theta_{f}\right)\right]^{1 / 2},
\end{aligned}
$$




$$
\frac{\partial^{2} S(r, \theta, E, \beta)}{\partial(r, \theta) \partial(E, \beta)}=-\frac{r}{2 \sin \theta_{f}} \frac{\partial \cos \theta_{f}}{\partial \beta} .
$$

Using our earlier assumptions, we can take $\beta=\cos \theta_{f}$ $=\cos \theta_{i} \equiv \cos \theta_{0}$. This way we get $\partial \beta / \partial q_{0}=-\sin \theta_{f}$. The substitution of this formula and Eq. (E11) into Eq. (D10) gives us

$$
\begin{aligned}
\operatorname{det}\left[J_{t}\left(r, \theta ; r_{0}, \theta_{0}\right)\right] & =-\frac{r^{2} \sin \theta \frac{\partial^{2} S_{\mathbf{M}}}{\partial \beta^{2}} \frac{\partial \beta}{\partial q_{0}}}{\operatorname{det}\left[\frac{\partial^{2} S}{\partial(r, \theta) \partial(E, \beta)}\right]} \\
& =-\frac{2 \sin ^{2} \theta_{f}}{r} \frac{\partial^{2} S_{\mathbf{M}}}{\partial \beta^{2}}\left(r^{2} \sin \theta\right) .
\end{aligned}
$$

Combining Eqs. (E8) and (E12), we obtain

$$
A_{2}\left(r_{0}, \theta_{f}\right)=\frac{\left(r_{0} / 2\right)^{1 / 4}}{\sin \theta_{f}}\left|\frac{\partial^{2} S}{\partial \beta^{2}}\right|^{-1 / 2} .
$$

The substitution of this expression for amplitude into Eqs. (E3) and (E2) gives the result

$$
C=2^{9 / 2} \pi^{3 / 2}\left(E-E_{i}\right)\left|\mathcal{Y}\left(\theta_{f}\right)\right|^{2}\left|\frac{\partial^{2} S}{\partial \beta^{2}}\right|^{-1 / 2} .
$$

This is equivalent to Eq. (60) for cylindrically symmetric systems, which confirms the agreement between two forms of semiclassical amplitudes for two-dimensional regular system.

In the three-dimensional case, it is convenient to take $\boldsymbol{\beta}$ $=\left(\cos \theta_{f}, \phi_{f}\right)$; after that we can find $\partial(\mathbf{a}) / \partial(\boldsymbol{\beta})=r^{2}$. Another determinant we need is equal to

$$
\operatorname{det}\left[\frac{\partial^{2} S}{\partial\left(E, \cos \theta_{f}, \phi_{f}\right) \partial(\mathbf{r})}\right]=-\frac{1}{2^{3 / 2} r_{0}^{1 / 2}}
$$

We get from Eq. (E9)

$$
A_{3}\left(\mathbf{q}, \mathbf{q}_{0}\right)=\left(\frac{r_{0}}{2}\right)^{1 / 2}\left|\operatorname{det}\left(\frac{\partial^{2} S}{\partial \beta_{i} \partial \beta_{j}}\right)\right|^{-1 / 2}
$$

This formula may be useful in three-dimensional applications of closed-orbit theory (such as an atom in crossed electric and magnetic fields).
[1] M.C. Gutzwiller, J. Math. Phys. 11, 1791 (1970).

[2] M.L. Du and J.B. Delos, Phys. Rev. A 38, 1896 (1988); 38, 1913 (1988). See also E.B. Bogomolny, Pis'ma Zh. Éksp. Teor. Fiz. 45, 445 (1988) [JETP Lett. 47, 526 (1988)]; D. Wintgen and H. Friedrich, Phys. Rev. A 36, 131 (1987).

[3] S.C. Creagh and N.D. Whelan, Phys. Rev. Lett. 77, 4975 (1996). They extended the results to include resonance widths in chaotic systems in the papers Ann. Phys. (Leipzig) 272, 196 (1999); Phys. Rev. Lett. 82, 5237 (1999). Their study of tunneling in a mixed phase space system (e-print chao-dyn/9910021) shows regular structure similar to that presented in our paper [4] and discussed in detail here.

[4] M.W. Beims, V. Kondratovich, and J.B. Delos, Phys. Rev. Lett. 81, 4537 (1998).

[5] E.B. Bogomolny and D.C. Rouben, Europhys. Lett. 43, 111 (1998); Eur. Phys. J. B 9, 695 (1999).

[6] D.S. Saraga and T.S. Monteiro, Phys. Rev. Lett. 81, 5796 (1998).

[7] M.V. Berry and M. Tabor, Proc. R. Soc. London, Ser. A 349, 101 (1976).

[8] M.C. Gutzwiller, Chaos in Classical and Quantum Mechanics (Springer-Verlag, Berlin, 1990); B. Hupper, J. Main, and G. Wunner, Phys. Rev. A 53, 744 (1996); G.S. Ezra, K. Richter, K. Tanner, and D. Wintgen, J. Phys. B 24, L413 (1991); N.L. Balazs and A. Voros, Phys. Rep. 143, 109 (1986).

[9] P.J. Redmond, Phys. Rev. 133, B1352 (1964).

[10] V. Kondratovich and J.B. Delos, Phys. Rev. A 57, 4654 (1998).
[11] V. Kondratovich and J.B. Delos, Phys. Rev. A 56, R5 (1997).

[12] We spell this out because other possibilities might also come to mind. For example, one could hold $\varepsilon$ real, allow $w$ to become complex, and weight the $\mathbf{n}$ th state by the imaginary part of $w_{\mathbf{n}}$. This is a possible definition, but the imaginary part of $w$ does not have a simple physical interpretation. In our definition [Eq. (25)] $w_{\mathbf{n}}$ is real and represents the location of each narrow resonance in a measured scaled spectrum, and $\hbar / \Gamma_{\mathbf{n}}$ represents the measurable lifetime for each decay by tunneling.

[13] M.S. Child, Semiclassical Mechanics with Molecular Applications (Oxford University Press, New York, 1991).

[14] For the spectrum of quasidiscrete states shown in Fig. 4(a), there is also a width related to decay by photon emission, but this is not the width considered in this paper.

[15] We integrate Eq. (31) using Taylor expansion of the argument of complex exponent. This works best when $K(\beta)$ varies rapidly, so the spectrum is dominated by states nearest the end point, i.e., the extremal torus. Otherwise, one should take into account the contributions of stationary points, as was pointed in the Introduction. It is easy to calculate in our case that the variation of the barrier transparency between the neighboring states of a Stark manifold is equal to $\delta K \approx 2 \pi \tau_{K} / \tau_{u}$. This quantity is easy to estimate for the red (downhill) edge of a manifold at the saddle energy $\varepsilon=-2$. Here the motion in the $u$ coordinate and below-barrier "motion" are almost harmonic with the ratio of periods equal to $\tau_{K} / \tau_{u}=\sqrt{2}$. Such a huge difference $\delta K=2^{3 / 2} \pi \approx 8.9$ is the reason that we see only the widths of the red states in the linear plot Fig. 6(b) [compare 
with the logarithmic plot Fig. 6(a)]. Above the saddle the $u$ motion on the extremal torus becomes anharmonic and the ratio $\tau_{K} / \tau_{u}$ diminishes. That is why we can see more states in the linear plot at $\varepsilon=-1.5$ [Fig. 6(b)].

[16] J. Gao and J.B. Delos, Phys. Rev. A 56, 356 (1997).

[17] P.J. Richens, J. Phys. A 15, 2101 (1982).

[18] At the energies near field-free ionization threshold $E=0$, the term $D^{+1} f(E)$ may lead in the orbital sum-see J. Gao and J.B. Delos, Phys. Rev. A 46, 1455 (1992).
[19] V. Kondratovich and J.B. Delos, Phys. Rev. A 57, 4604 (1998).

[20] We also have to account for the parallel orbits and repair their bifurcations. Full details on this aspect of the calculation will appear in a later publication.

[21] V.P. Maslov and M. Fedoriuk, Semiclassical Approximation in Quantum Mechanics (Reidel, Boston, 1981); see also the discussion of this method in J.B. Delos, Adv. Chem. Phys. 65, 161 (1986). 